\title{
EXTENSION OF CR STRUCTURES ON PSEUDOCONVEX CR MANIFOLDS WITH ONE DEGENERATE EIGENVALUE
}

\author{
SANGHYUN CHO
}

(Received June 20, 2001, revised February 12, 2002)

\begin{abstract}
Let $\bar{M}$ be a smoothly bounded orientable pseudoconvex CR manifold of finite type with at most one degenerate eigenvalue. Then we extend the given CR structure on $M$ to an integrable almost complex structure on the concave side of $M$. Therefore we may regard $M$ as the boundary of a complex manifold.
\end{abstract}

1. Introduction. Suppose that $\bar{M}$ is an abstract smoothly bounded orientable CR manifold of dimension $2 n-1$ with a given integrable CR structure $\mathcal{S}$ of dimension $n-1$. Since $\bar{M}$ is orientable, there are a smooth real nonvanishing 1-form $\eta$ and a smooth real vector field $X_{0}$ on $\bar{M}$ so that $\eta(X)=0$ for all $X \in \mathcal{S}$ and $\eta\left(X_{0}\right)=1$. We define the Levi form of $\mathcal{S}$ on $\bar{M}$ by $i \eta\left(\left[X^{\prime}, \bar{X}^{\prime \prime}\right]\right), X^{\prime}, X^{\prime \prime} \in \mathcal{S}$. We may assume that $\bar{M} \subset \tilde{M}$, in $C^{\infty}$ sense, where $\tilde{M}$ is a smooth manifold.

In [5], Catlin has studied an extension problem of a given CR structure on $M$ to an integrable almost complex structure on a $2 n$-dimensional manifold $\Omega$ with boundary $b \Omega$, so that the extension is smooth up to the boundary and so $M$ lies in $b \Omega$. Under certain conditions on the Levi form (cf. [5, Theorem 1.1, Theorem 1.3]), this shows that an abstract CR manifold can be locally embedded in $\boldsymbol{C}^{n}[1,13,16]$.

In this paper, we study an extension problem of a given CR structure on $M$ when $M$ is a pseudoconvex CR manifold of finite type with one degenerate eigenvalue and $\operatorname{dim}_{\boldsymbol{R}} M=$ $2 n-1$. For a given positive continuous function $g$ on $M$, where $g=0$ on $b M$, the boundary of $M$, we define

$$
S_{g}^{+}=\{(x, t) \in M \times[0, \infty) ; 0 \leq t \leq g(x)\} .
$$

Then our main result is the following theorem:

THEOREM 1.1. Let $(\bar{M}, \mathcal{S})$ be a smoothly bounded pseudoconvex CR manifold of finite type with at most one degenerate eigenvalue and $\operatorname{dim}_{R} M=2 n-1$. Then there exists a positive continuous function $g$ on $M$ and a smooth integrable almost complex structure $\mathcal{L}$ on $S_{g}^{+}$such that for all $x \in M, \mathcal{L}_{(x, 0)} \cap C T M=\mathcal{S}_{x}$. Furthermore, if $\mathcal{J}_{\mathcal{L}}: T S_{g}^{+} \rightarrow T S_{g}^{+}$ is the map associated with the complex structure $\mathcal{L}$, then $d t\left(\mathcal{J}_{\mathcal{L}}\left(X_{0}\right)\right)<0$ at all points of $M_{0}=\{(x, 0) ; x \in M\}$.

2000 Mathematics Subject Classification. Primary 32G05; Secondary 32 GO7.

Key words and phrases. CR manifold, finite type, pseudoconvex.

Partially supported by KOSEF 971-0102-011-2 and by R01-1999-00000 from KOSEF. 
Note that we extend the given CR structure on $M$ to the concave side (instead of convex side) of $M$. When $\operatorname{dim}_{R} M=3$, the author proved the same result when $\bar{M}$ is a pseudoconvex CR manifold of finite type [8]. We also note that if $M$ is strongly pseudoconvex, this case was handled in [5, Theorem 1.1].

In general, Theorem 1.1 does not imply that the given CR structure can be locally embedded in $\boldsymbol{C}^{n}$ [12]. On the other hand, a theorem of Newlander-Nirenberg [14] shows that an integrable almost complex manifold is a complex manifold. Therefore, if we let $(M, \mathcal{S})$ be as in Theorem 1.1, then we have the following corollaries.

COROLlARY 1.2. We may regard $(M, \mathcal{S})$ as a boundary of a complex manifold.

COROLlary 1.3. Let $D$ be a complex manifold with $C^{\infty}$ boundary $b D$, and $\operatorname{dim}_{C} D=$ $n$. Suppose that the almost complex structure on $D$ extends smoothly to a manifold $\bar{M} \subset b D$, where $\bar{M}$ is a smoothly bounded pseudoconvex CR manifold of finite type and the Levi-form of $\bar{M}$ has one degenerate eigenvalue and $\operatorname{dim}_{R} M=2 n-1$. Then $D$ can be embedded into a larger complex manifold $\Omega$ so that $M$ lies in the interior of $\Omega$ as a real hypersurface.

REMARK 1.4. In [6], the author showed that any smooth compact pseudoconvex complex manifold $\bar{D}$ of finite type with $\operatorname{dim}_{C} D=n, n \geq 2$, can be embeded into a larger complex manifold $\Omega$. Corollary 1.3 is a generalization of this result to some special non-compact complex manifolds.

REMARK 1.5. If $(M, \mathcal{S})$ has at least three positive eigenvalues, Catlin [5] has extended the given CR structure of $\mathcal{S}$ to the pseudoconvex side of $\bar{M}$ [5, Theorem 1.1]. If $M$ is also pseudoconvex, this result implies the local embedding of CR structures in $\boldsymbol{C}^{n}$.

In [5], Catlin has introduced certain nonlinear equations which stem from deformation theory of an almost complex structure (Section 2). The linearized forms of these equations are simply the $\bar{\partial}$-operator from $\Lambda^{0,1} \otimes T^{1,0}$ to $\Lambda^{0,2} \otimes T^{1,0}$ (Section 2). To overcome difficulties in subelliptic estimates for $\bar{\partial}$ near $b M$, we choose a Hermitian metric on $S_{g}^{+}$so that $S_{g}^{+}$takes on the form $S_{\varepsilon}=M \times[0, \varepsilon]$. To this end, we choose, for each $x_{0} \in M$, a noneuclidean ball that is of size $\delta=g\left(x_{0}\right)$ in the transverse holomorphic direction and of size $\delta^{1 / 2}$ in strongly pseudoconvex tangential holomorphic directions, and of size $\tau\left(x_{0}, \delta\right)$ in the weakly pseudoconvex tangential holomorphic direction. We choose the metric so that the unit ball about $x_{0} \in M$ corresponds to the above noneuclidean ball with $\delta=g\left(x_{0}\right)$.

To show that $\tau\left(x_{0}, \delta\right)$ is invariantly defined (i.e., independent of coordinate functions), we choose special coordinates defined near $x_{0} \in M$ (Proposition 3.1). These change of coordinates shall have an independent interest in studying weakly pseudoconvex CR manifolds of finite type. In terms of these special coordinates, the weakly pseudoconvex tangential holomorphic vector field $L_{1}$ has a special representation so that we can define another quantity $\mu(x, \delta)$, which is a smooth function of $\delta$ and $x$ and it is obtained by taking sucessive brackets of $L_{1}$ and $\bar{L}_{1}$ and hence defined invariantly. A technical difficulty is to show that the brackets mixed with $L_{1}$ and the strongly pseudoconvex tangential vector fields are not major terms in determining $\mu(x, \delta)$. Then we show that $\tau(x, \delta) \approx \mu(x, \delta)$ (Proposition 3.5), and hence 
$\tau(x, \delta)$ is defined invariantly. To get an $0<\varepsilon \leq 1 / 2$ subelliptic estimates (other than $1 / 2$ estimates of Catlin [5]), we also need some precise estimates for $\eta\left(\left[L_{1}, \bar{L}_{k}\right]\right), 2 \leq k \leq n$. This difficulty comes from the fact that we have a Dirichlet condition on $M_{0}$ and hence we need to control the boundary integral terms, which were not occured in dimension three case, on $M_{0}$ (In usual $\bar{\partial}$-estimates we deal with forms which vanish on $M_{0}$ and hence there are no boundary integral terms). Section 3, 4 and 5 contain these estimates in detail.

After this, we technically construct a family of plurisubharmonic functions with large Hessian using the properties of $\tau(x, \delta)$ and $\mu(x, \delta)$. In performing the subelliptic estimates, we use $(n-2)$-positive eigenvalue conditions on $M_{\sigma}$ to handle the boundary integral terms on $M_{\sigma}$, and we use the existance of a family of plurisubharmonic functions with large Hessian to handle the components vanishing on $M_{\sigma}$. This will give us uniform $1 / m$ subelliptic estimates for $\bar{\partial}$ on each non-euclidean ball. Then we get the estimates, so-called "tame estimates", which are required in the Nash-Moser theorem [14] for the approximate solution to the linearized equation.

2. Deformation of almost complex structures. Let $(M, \mathcal{S})$ be a CR manifold as in Section 1 and set $\Omega=M \times(-1,1)$. In this section we extend the given CR structure $\mathcal{S}$ on $M$ to an almost complex manifold $(\Omega, \mathcal{L})$, and study a deformation problem of the almost complex structure $\mathcal{L}$ on $\Omega$ so that the new (deformed) amost complex structure is integrable (or close to be integrable).

Assume that $\mathcal{L}$ is an almost complex structure on $\Omega$. Let $A$ be a smooth section of $\Gamma^{1}(\mathcal{L})=\Lambda^{0,1}(\mathcal{L}) \otimes \mathcal{L}$, where $\Lambda^{0,1}(\mathcal{L})$ denotes the set of $(0,1)$ forms with respect to $\mathcal{L}$. Observe that if $A$ is sufficiently small, then the bundle $\mathcal{L}^{A}=\{L+\bar{A}(L) ; L \in \mathcal{L}\}$ defines a new almost complex structure and if $\bar{L}^{\prime}$ and $\bar{L}^{\prime \prime}$ are sections of $\overline{\mathcal{L}}$, then $\bar{L}^{\prime}+A\left(\bar{L}^{\prime}\right)$ and $\bar{L}^{\prime \prime}+A\left(\bar{L}^{\prime \prime}\right)$ are sections of $\overline{\mathcal{L}^{A}}$. Similarly, if $\omega$ is a section of $\Lambda^{1,0}(\mathcal{L})$, then $\omega-A^{*} \omega$ is a section of $\Lambda^{1,0}\left(\mathcal{L}^{A}\right)$, where the adjoint $A^{*}$ maps from $\Lambda^{1,0}(\mathcal{L})$ to $\Lambda^{0,1}(\mathcal{L})$ and is defined by

$$
\left(A^{*} \omega\right)(\bar{L})=\omega(A(\bar{L}))
$$

for all $\bar{L} \in \overline{\mathcal{L}}$ and $\omega \in \Lambda^{1,0}$. We want to choose $A$ so that

$$
\left(\omega-A^{*} \omega\right)\left(\left[L^{\prime}+A\left(L^{\prime}\right), L^{\prime \prime}+A\left(L^{\prime \prime}\right)\right]\right)=0 .
$$

By linearizing, i.e., by ignoring terms where $A$ or $A^{*}$ appear more than once, we obtain

$$
\omega\left(\left[L^{\prime}, A\left(L^{\prime \prime}\right)\right]\right)+\omega\left(\left[A\left(L^{\prime}\right), L^{\prime \prime}\right]\right)-A^{*} \omega\left(\left[L^{\prime}, L^{\prime \prime}\right]\right)=-\omega\left(\left[L^{\prime}, L^{\prime \prime}\right]\right) .
$$

Let $L=L^{\prime}+L^{\prime \prime}$ denote the decomposition of a vector $L \in C T_{z}$ where $L^{\prime} \in \mathcal{L}_{z}$ and $L^{\prime \prime} \in \overline{\mathcal{L}}_{z}$. For sections $\bar{L}_{1}, \bar{L}_{2}$ of $\overline{\mathcal{L}}$, we define

$$
\left(D_{2} A\right)\left(\bar{L}_{1}, \bar{L}_{2}\right)=\left[\bar{L}_{1}, A\left(\bar{L}_{2}\right)\right]^{\prime}-\left[\bar{L}_{2}, A\left(\bar{L}_{1}\right)\right]^{\prime}-A\left(\left[\bar{L}_{1}, \bar{L}_{2}\right]^{\prime \prime}\right) .
$$

Note that this definition is linear in $\bar{L}_{1}$ and $\bar{L}_{2}$, and hence $D_{2} A$ is a section of $\Gamma^{2}=\Lambda^{0,2}(\mathcal{L}) \otimes$ $\mathcal{L}$. It follows from (2.1) and (2.3) that (2.2) is equivalent to the equation

$$
D_{2} A=-F,
$$


where $F$ is a section of $\Gamma^{2}$ defined by

$$
F\left(\bar{L}_{1}, \bar{L}_{2}\right)=\left[\bar{L}_{1}, \bar{L}_{2}\right]^{\prime} .
$$

Note that $F$ measures to what extent $\mathcal{L}$ fails to be integrable. If $\mathcal{L}$ defines a CR structure on $M$ and if we want $\mathcal{L}_{A}$ to define the same CR structure on $M$, then this means that $A$ must satisfy $A\left(\bar{L}^{\prime}\right)=0$ on $M$ whenever $\bar{L}^{\prime}$ is a section of $\overline{\mathcal{L}}$ that is tangent to $M$. This is a Dirichlet condition on some of the components of the solution (2.4).

If we define $D_{3}: \Gamma^{2} \rightarrow \Gamma^{3}=\Lambda^{0,3}(\mathcal{L}) \otimes \mathcal{L}$ by

$$
\begin{aligned}
D_{3} B\left(\bar{L}_{1}, \bar{L}_{2}, \bar{L}_{3}\right)= & {\left[\bar{L}_{1}, B\left(\bar{L}_{2}, \bar{L}_{3}\right)\right]^{\prime}-\left[\bar{L}_{2}, B\left(\bar{L}_{1}, \bar{L}_{3}\right)\right]^{\prime}+\left[\bar{L}_{3}, B\left(\bar{L}_{1}, \bar{L}_{2}\right)\right]^{\prime} } \\
& -B\left(\left[\bar{L}_{1}, \bar{L}_{2}\right]^{\prime \prime}, \bar{L}_{3}\right)+B\left(\left[\bar{L}_{1}, \bar{L}_{3}\right]^{\prime \prime}, \bar{L}_{2}\right)-B\left(\left[\bar{L}_{2}, \bar{L}_{3}\right]^{\prime \prime}, \bar{L}_{1}\right)
\end{aligned}
$$

for $B \in \Gamma^{2}$, then it follows that $D_{3} F=0$ [5, Lemma 3.2]. Then we have the following formal solution of the extension problem [5, Theorem 4.1].

THEOREM 2.1. Suppose that $M$ is an orientable CR manifold of dimension $2 n-1$ such that the CR dimension equals $n-1$. Then there exists an almost complex structure $\mathcal{L}^{*}$ on $\Omega=M \times(-1,1)$ such that $\mathcal{L}^{*}$ is an extension of the $C R$ structure on $M$, and such that it is integrable to infinite order at $M$ in the sense that if $\omega$ is a section of $\Lambda^{1,0}\left(\mathcal{L}^{*}\right)$ and $\bar{L}_{1}, \bar{L}_{2}$ are sections of $\overline{\mathcal{L}}^{*}$, then $\omega\left(\left[\bar{L}_{1}, \bar{L}_{2}\right]\right)$ vanishes to infinite order along $M$.

Let $M$ and $\Omega$ be as in Theorem 2.1. The next theorem shows that the above formal extension is essentially unique [5, Theorem 4.2].

THEOREM 2.2. Suppose that $\mathcal{L}$ and $\mathcal{X}$ are almost complex structures on $\Omega$ that extend the CR-structure on $M_{0}=\{(x, 0) ; x \in M\}$, and that are integrable to infinite order on $M_{0}$. Then there exists a diffeomorphism $G$ of $\Omega$ onto itself that is the identity when $t=0$ and such that $G_{*} \mathcal{X}$ approximates $\mathcal{L}$ to infinite order near $M_{0}$ in the sense that if $X$ is a section of $\mathcal{L}$, then $G_{*} X$ differs from a section of $\mathcal{L}$ by a vector field which vanishes to infinite order on $M_{0}$.

By Theorem 2.1, we have an almost complex structure $\mathcal{L}^{*}$ on $\Omega$, that is integrable to infinite order along $M_{0}=\{(x, 0) ; x \in M\}$. Let $\eta$ be a smooth non-vanishing one form on $M$ that satisfies $\eta(L)=0$ for all $L \in \mathcal{S}_{x}, x \in M$, and that defines the Levi form of $M$ as in Section 1. We can clearly extend $\eta$ to all of $\Omega$ so that it still annihilates $\mathcal{S}_{(x, t)}$ for all $(x, t) \in \Omega$, where $\mathcal{S}_{(x, t)}$ now denotes the space of vectors in $\mathcal{L}_{(x, t)}^{*}$ that are tangent to the level set of the auxiliary coordinate $t$. Then we have the following theorem which is a formal solution of local embedding problem. One can refer a proof to, for example, [3, Proposition 3].

THEOREM 2.3. Let $x_{0} \in M$. Then there are a small neighborhood $U$ of $x_{0}$ and a constant $c>0$ so that for each $x \in M \cap U$, there are almost holomorphic functions $f_{1}, \ldots, f_{n}$ defined on $\bar{U}$ so that if $F_{x}=\left(f_{1}, \ldots, f_{n}\right)$, then $F_{x}(x)=0$ and

(a) $\left|d F_{x}\right| \geq c$ on $\bar{U}$, and

(b) $\bar{L} f_{j}$ vanishes to infinite order at $x_{0}$ for each $L \in \mathcal{L}$. 
REMARK 2.4. Suppose $L \in \mathcal{S}_{(x, 0)}$. Then $\left(F_{x_{0}}\right)_{*} L$ differs from a section of $T^{1,0}\left(F_{x_{0}}(M)\right)$ by a vector field which vanishes to infinite order at 0 . Therefore the image $F_{x_{0}}(M)$ is a smooth real hypersurface in $\boldsymbol{C}^{n}$ with defining function given by $r(w)=t \circ F_{x_{0}}^{-1}(w)$.

In order to define the type of $x_{0} \in M$, we use the (almost) holomorphic function $F_{x}$ constructed in Theorem 2.3.

Definition 2.5. Let $\left(x_{0}, U, F_{x_{0}}\right)$ be as in Theorem 2.3. Then we define the type of $x_{0}$ is equal to the type of $F_{x_{0}}\left(x_{0}\right)=0 \in \boldsymbol{C}^{n}$ in the sense of D'Angelo [9].

Set $T\left(x_{0}\right)=$ the type of $x_{0} \in M$, and set

$$
T(\bar{M})=\max \left\{T\left(x_{0}\right) ; x_{0} \in \bar{M}\right\}=m .
$$

Under the assumption that the Levi-form of $M$ has $(n-2)$-positive eigenvalues, we may assume that $m$ is an even integer.

Let us take $\left(\Omega, \mathcal{L}^{*}\right)$ constructed in Theorem 2.1. Choose a smooth real vector field $X_{0}$ on $\Omega$ that satisfies $X_{0} t \equiv 0$ and $\eta\left(X_{0}\right) \equiv 1$ in $\Omega$. Set $Y_{0}=-\mathcal{J}_{\mathcal{L}^{*}}\left(X_{0}\right)$ so that $X_{0}+i Y_{0}$ is a section of $\mathcal{L}^{*}$ that is transverse to the level set of $t$. Let $G: \Omega \rightarrow \Omega$ be a diffeomorphism such that $G$ fixes $M_{0}$ and

$$
G_{*} Y_{\left.0\right|_{(x, 0)}}=\left.\frac{\partial}{\partial t}\right|_{(x, 0)}, \quad x \in M .
$$

Since $M$ is orientable, we may assume that $d t\left(\mathcal{J}_{\mathcal{L}^{*}}\left(X_{0}\right)\right)<0$. Thus $d t\left(Y_{0}\right)>0$ along $M_{0}$, which shows that $G$ preserves the sides of $M_{0}$, i.e., $G$ maps $\Omega^{+}=\{(x, t) ; 0 \leq t<1\}$ into itself. If we set $\mathcal{L}^{0}=G_{*} \mathcal{L}^{*}$, then clearly $\tilde{Z}=-i G_{*}\left(X_{0}+i Y_{0}\right)$ is a section of $\mathcal{L}^{0}$ such that along $M_{0}$,

$$
\tilde{Z}=-i X_{0}+\frac{\partial}{\partial t}
$$

We write $\tilde{Z}=\tilde{X}+g(x, t) \partial / \partial t$ where $\tilde{X} t \equiv 0$, and set $L_{n}=g^{-1} \tilde{Z}$. Then $L_{n}=\partial / \partial t+X$, where $X t \equiv 0$. We fix a smooth metric $\langle,\rangle_{0}$ that is Hermitian with respect to the structure $\mathcal{L}^{0}$ on $\Omega$. Note that along $M$, we have $L_{n}=\partial / \partial t-i X_{0}$ and $d t=(1 / 2)(d t+i \eta)+(1 / 2)(d t-i \eta)$, which implies that $\partial t=(1 / 2)(d t+i \eta)$. Hence $\partial t(L)=(1 / 2) d t(L)+(i / 2) \eta(L)$ and

$$
\partial t\left(\left[X_{1}, \bar{X}_{2}\right]\right)=\frac{i}{2} \eta\left(\left[X_{1}, \bar{X}_{2}\right]\right)
$$

for all $X_{1}, X_{2} \in \mathcal{S}_{(x, t)}$, along $M$.

3. Construction of plurisubharmonic functions. Let $M, \Omega, X_{0}$ and $\mathcal{L}^{0}$ be as in Section 2. In this section, we will construct a family of plurisubharmonic functions with large Hessian (Theorem 3.6). For this purpose, we first construct special coordinate functions defined in a neighborhood of $x_{0} \in M$ so that the coefficients of the weakly pseudoconvex tangential holomorphic vector fields satisfy some necessary estimates in new coordinates (Lemma 3.7 and Proposition 3.8). 
Assume that $\tilde{x}_{0} \in M$. Then there are coordinate functions $x_{1}, \ldots, x_{2 n}$ defined on a neighborhood $\bar{U}$ of $\tilde{x}_{0}$ with the property that $x_{2 n}=t$ and that

$$
x_{k}\left(x^{\prime}, t\right)=x_{k}\left(x^{\prime}, 0\right), \quad k<2 n,
$$

for $\left(x^{\prime}, t\right) \in \bar{U}$, and that

$$
\frac{\partial}{\partial x_{2 n-1}}=-X_{0}
$$

at all points of $\bar{U} \cap M$. We take an orthonormal frame $\left\{\tilde{L}_{1}, \ldots, \tilde{L}_{n}\right\}$ of $\mathcal{L}^{0}$ defined on $\bar{U}$. Let $x_{0} \in M \cap U$ be fixed for a moment. If $\tilde{L}_{j}$ is replaced by $L_{j}=\sum_{k=1}^{n-1} U_{j k} \tilde{L}_{k}$ where $U=\left(U_{j k}\right)$ is a suitably chosen unitary matrix so that

$$
\frac{i}{2} \eta\left(\left[L_{j}, \bar{L}_{k}\right]\right)\left(x_{0}\right)=\delta_{j k} d_{j}\left(x_{0}\right):=d_{j, k}\left(x_{0}\right), \quad 1 \leq j, k \leq n-1,
$$

where $0 \leq d_{1} \leq d_{2} \leq \cdots \leq d_{n-1}$, and $d_{j}\left(x_{0}\right)$ is a smooth function defined on $\bar{U}$ satisfying $d_{2}(x) \geq d_{0}>0$ on $\bar{U}$ for a uniform constant $d_{0}>0$, while $\delta_{j k}$ is a Dirac delta function.

There is an affine transformation $C_{x_{0}}: \boldsymbol{R}^{2 n} \rightarrow \boldsymbol{R}^{2 n}$ such that if $\left(x^{\prime}, 0\right) \in \boldsymbol{R}^{2 n}$ are the coordinates of $x \in M$, then

$$
C_{x_{0}}\left(x^{\prime}, t\right)=\left(P_{x_{0}}\left(x^{\prime}-x_{0}^{\prime}\right), t\right):=\left(u_{1}, \ldots, u_{2 n}\right)=u,
$$

where the $(2 n-1) \times(2 n-1)$ constant matrix $P_{x_{0}}$ is chosen so that in terms of $u$-coordinates,

$$
\begin{aligned}
& \left.L_{k}\right|_{x_{0}}=\frac{\partial}{\partial u_{2 k-1}}-i \frac{\partial}{\partial u_{2 k}}, \quad 1 \leq k \leq n, \\
& \left.X_{0}\right|_{x_{0}}=-\frac{\partial}{\partial u_{2 n-1}} .
\end{aligned}
$$

Note that the second equality of (3.2) actually implies that $\left.X_{0}\right|_{\left(x^{\prime}, 0\right)}=-\partial / \partial u_{2 n-1}$ at all points of $M \cap \bar{U}$, and hence that

$$
L_{n}=-i \frac{\partial}{\partial u_{2 n-1}}+\frac{\partial}{\partial t}
$$

along $M \cap \bar{U}$.

We also note that the matrix $P_{x_{0}}$ is uniquely determined by the condition (3.2) and uniformly non-singular on $\bar{U}$, and depends smoothly for all $x_{0} \in M \cap \bar{U}$. In terms of $u$ coordinates, the vector fields $L_{k}, 1 \leq k \leq n-1$, can be written as

$$
\begin{aligned}
L_{k}= & \left(\frac{\partial}{\partial u_{2 k-1}}+\sum_{j=1}^{2 n-2} a_{j}^{k}(u) \frac{\partial}{\partial u_{j}}+a_{k}(u) \frac{\partial}{\partial u_{2 n-1}}\right) \\
& -i\left(\frac{\partial}{\partial u_{2 k}}+\sum_{j=1}^{2 n-2} b_{j}^{k}(u) \frac{\partial}{\partial u_{j}}+b_{k}(u) \frac{\partial}{\partial u_{2 n-1}}\right),
\end{aligned}
$$

where $a_{j}^{k}, b_{j}^{k}, a_{k}, b_{k}$ are smooth real valued functions which vanish at 0 . In the sequal we let $\partial_{l}, 1 \leq l \leq n$, denote the holomorphic partial derivatives in the $l$-th variable of local complex 
valued coordinates. When we change the local coordinate functions, this partial derivative operator will be written in new coordinates. We also let $\tilde{\partial}_{\beta}$ denotes $\partial_{\beta}$ or $\bar{\partial}_{\beta}$.

PROPOSITION 3.1. For each $x_{0} \in M \cap U$ and positive integer $m$, there are smooth complex valued coordinates $\zeta=\left(\zeta_{1}, \ldots, \zeta_{n}\right), \zeta_{n}=t+i x_{2 n-1}$, defined near $x_{0}$ such that in $\zeta$-coordinates, the vector fields $L_{1}, \ldots, L_{n-1}$ can be written as

$$
\begin{aligned}
& L_{1}=\frac{\partial}{\partial \zeta_{1}}+\sum_{l=1}^{n-1} a_{l}(\zeta) \frac{\partial}{\partial \zeta_{l}}+\sum_{l=1}^{n-1} b_{l}(\zeta) \frac{\partial}{\partial \bar{\zeta}_{l}}+(e(\zeta)+i d(\zeta)) \frac{\partial}{\partial x_{2 n-1}}, \\
& L_{\alpha}=\frac{\partial}{\partial \zeta_{\alpha}}+\sum_{l=1}^{n-1} a_{l}^{\alpha}(\zeta) \frac{\partial}{\partial \zeta_{l}}+\sum_{l=1}^{n-1} b_{l}^{\alpha}(\zeta) \frac{\partial}{\partial \bar{\zeta}_{l}}+\left(e_{\alpha}(\zeta)+i d_{\alpha}(\zeta)\right) \frac{\partial}{\partial x_{2 n-1}}
\end{aligned}
$$

where $2 \leq \alpha \leq n-1$. Also, the coefficient functions satisfy

$$
\begin{aligned}
& \partial_{1}^{j} \bar{\partial}_{1}^{k} b_{l}(0)=\partial_{1}^{j} \bar{\partial}_{1}^{k} a_{l}^{\alpha}(0)=\partial_{1}^{j} \bar{\partial}_{1}^{k} b_{l}^{\alpha}(0)=0, \quad j+k \leq m, \quad 2 \leq l \leq n-1, \\
& \tilde{\partial}_{\beta}^{i} \partial_{1}^{j} \bar{\partial}_{1}^{k} e(0)=0, \quad i=0,1, \quad i+j+k \leq m, \quad 2 \leq \beta \leq n-1, \\
& \left(\partial_{1}-\bar{\partial}_{1}\right)^{s} d(0)=\left(\partial_{1}-\bar{\partial}_{1}\right)^{s} e_{\alpha}(0)=\left(\partial_{1}-\bar{\partial}_{1}\right)^{s} d_{\alpha}(0)=0, \quad s \leq m .
\end{aligned}
$$

Proof. Let us take the vector fields $L_{1}, \ldots, L_{n}$ and smooth coordinates $u$ defined near $x_{0}$ so that the vector fields $L_{k}, 1 \leq k \leq n-1$, have the representation as in (3.3). Therefore (3.4) and (3.5) hold for $m=p=0$.

Assume by induction that there are smooth complex valued coordinates $\zeta=\left(\zeta_{1}, \ldots, \zeta_{n}\right)$ defined near $x_{0} \in M$ so that in terms of $\zeta$-coordinates, we can write the vector fields $L_{v}$, $1 \leq v \leq n-1$, as in (3.4) where the coefficients satisfy the estimates in (3.5) for $m$ replaced by $p \geq 0$. Set

$$
\begin{aligned}
& x_{2 j-1}=\zeta_{j}+\bar{\zeta}_{j}, x_{2 j}=-i\left(\zeta_{j}-\bar{\zeta}_{j}\right), \quad 1 \leq j<n, \\
& x_{2 n-1}=-i\left(\zeta_{n}-\bar{\zeta}_{n}\right), x_{2 n}=t
\end{aligned}
$$

and set

$$
D_{k}=\frac{\partial}{\partial x_{k}}, \quad 1 \leq k \leq 2 n-1
$$

In $x=\left(x_{1}, \ldots, x_{2 n-1}, t\right)$ coordinates, each vector field $L_{v}, 1 \leq v \leq n-1$, can be written as

$$
\begin{aligned}
L_{v}= & \left(\frac{\partial}{\partial x_{2 v-1}}+\sum_{l=1}^{2 n-2} c_{l}^{v}(x) \frac{\partial}{\partial x_{l}}+e_{\nu}(x) \frac{\partial}{\partial x_{2 n-1}}\right) \\
& -i\left(\frac{\partial}{\partial x_{2 v}}+\sum_{l=1}^{2 n-2} d_{l}^{v}(x) \frac{\partial}{\partial x_{l}}+d_{v}(x) \frac{\partial}{\partial x_{2 n-1}}\right)
\end{aligned}
$$


where $c_{l}^{v}, d_{l}^{v}, e_{v}, d_{v}$ are smooth real valued functions. Set

$$
\begin{aligned}
& \tilde{x}_{i}=x_{i}, \quad i=1,2, \\
& \tilde{x}_{l}=x_{l}-\sum_{\nu=2}^{n-1} \sum_{j+k=p+1} \frac{1}{j ! k !}\left[D_{1}^{j} D_{2}^{k} c_{l}^{\nu}(0) x_{1}^{j} x_{2}^{k} x_{2 \nu-1}+D_{1}^{j} D_{2}^{k} d_{l}^{v}(0) x_{1}^{j} x_{2}^{k} x_{2 \nu}\right]
\end{aligned}
$$

for $l=3, \ldots, 2 n-2$, and set

$$
\begin{aligned}
\tilde{x}_{2 n-1}= & x_{2 n-1}-\sum_{j+k=p+1} \frac{1}{(j+1) ! k !}\left[D_{1}^{j} D_{2}^{k} e_{1}(0) x_{1}^{j+1} x_{2}^{k}\right] \\
& -\sum_{\beta=3}^{2 n-2} \sum_{j+k=p} \frac{1}{(j+1) ! k !}\left[D_{\beta} D_{1}^{j} D_{2}^{k} e_{1}(0) x_{1}^{j+1} x_{2}^{k} x_{\beta}\right] \\
& -\sum_{\nu=2}^{n-1}\left[\frac{1}{(p+1) !} D_{2}^{p+1} e_{\nu}(0) x_{2}^{p+1} x_{2 v-1}+\frac{1}{(p+1) !} D_{2}^{p+1} d_{v}(0) x_{2}^{p+1} x_{2 v}\right] \\
& -\frac{1}{(p+2) !} D_{2}^{p+1} d_{1}(0) x_{2}^{p+2} .
\end{aligned}
$$

If we set

$$
w_{j}=\frac{1}{2}\left(\tilde{x}_{2 j-1}+i \tilde{x}_{2 j}\right), \quad 1 \leq j \leq n-1, \quad w_{n}=\frac{1}{2}\left(t+i \tilde{x}_{2 n-1}\right),
$$

then in $w$-coordinates, the vector fields $L_{\alpha}, 1 \leq \alpha \leq n-1$, can be written as

$$
\begin{aligned}
& L_{1}=\frac{\partial}{\partial w_{1}}+\sum_{l=1}^{n-1} \tilde{a}_{l}(w) \frac{\partial}{\partial w_{l}}+\sum_{l=1}^{n-1} \tilde{b}_{l}(w) \frac{\partial}{\partial \bar{w}_{l}}+(\tilde{e}(w)+i \tilde{d}(w)) \frac{\partial}{\partial \tilde{x}_{2 n-1}}, \\
& L_{\alpha}=\frac{\partial}{\partial w_{\alpha}}+\sum_{l=1}^{n-1} \tilde{a}_{l}^{\alpha}(w) \frac{\partial}{\partial w_{l}}+\sum_{l=1}^{n-1} \tilde{b}_{l}^{\alpha}(w) \frac{\partial}{\partial \bar{w}_{l}}+\left(\tilde{e}_{\alpha}(w)+i \tilde{d}_{\alpha}(w)\right) \frac{\partial}{\partial \tilde{x}_{2 n-1}}
\end{aligned}
$$

where for $2 \leq l, \alpha, \beta \leq n-1$, we have

$$
\begin{aligned}
& \partial_{1}^{j} \bar{\partial}_{1}^{k} \tilde{b}_{l}(0)=0, \tilde{\partial}_{\beta} \partial_{1}^{j} \bar{\partial}_{1}^{k} \tilde{e}(0)=0, \quad j+k \leq p, \\
& \partial_{1}^{j} \bar{\partial}_{1}^{k} \tilde{a}_{l}^{\alpha}(0)=\partial_{1}^{j} \bar{\partial}_{1}^{k} \tilde{b}_{l}^{\alpha}(0)=\partial_{1}^{j} \bar{\partial}_{1}^{k} \tilde{e}(0)=0, \quad j+k \leq p+1, \\
& \left(\partial_{1}-\bar{\partial}_{1}\right)^{s} \tilde{d}(0)=\left(\partial_{1}-\bar{\partial}_{1}\right)^{s} \tilde{e}_{\alpha}(0)=\left(\partial_{1}-\bar{\partial}_{1}\right)^{s} \tilde{d}_{\alpha}(0)=0, \quad s \leq p+1 .
\end{aligned}
$$

We perform the following change of coordinates:

$$
\begin{aligned}
& \zeta_{1}=w_{1}, \\
& \zeta_{l}=w_{l}-\sum_{j+k=p+1} \frac{1}{(j+1) ! k !}\left[\bar{\partial}_{1}^{j} \partial_{1}^{k} \overline{\tilde{b}}_{l}(0) \bar{w}_{1}^{j+1} w_{1}^{k}\right], \quad 2 \leq l \leq n-1 .
\end{aligned}
$$

Then in terms of $\zeta$-coordinates, we may write the vector fields $L_{1}, \ldots, L_{n-1}$ as in (3.4) and the coefficients of $L_{\alpha}, 1 \leq \alpha \leq n-1$, satisfy (3.5) where $m$ is replaced by $p+1$. If we proceed up to $m$-steps, we will get a proof of the proposition. 
For each $x_{0} \in M \cap U$ and $\delta>0$, we want to define a quantity $\tau\left(x_{0}, \delta\right)$ in such a way that the sucessive derivatives of the coefficients of $L_{k}, 1 \leq k \leq n-1$, up to a certain order (less than or equal to $m$ ), change by no more than $\delta$ on a nonisotropic ball about $x_{0}$. We use the special coordinates $\zeta=\left(\zeta_{1}, \zeta_{2}, \ldots, \zeta_{n}\right)$ defined near $x_{0}$ as in Proposition 3.1.

Assume $x_{0} \in M \cap U$. In terms of $\zeta$-coordinates, we write $L_{k}, 1 \leq k \leq n-1$, as in (3.4) such that the coefficient functions satisfy the estimates in (3.5). Note that we may assume that $m$ is an even integer. Let $b(\zeta)=e(\zeta)+i d(\zeta)$ be the coefficient function of $\partial / \partial x_{2 n-1}$ in $L_{1}$, and set

$$
b_{m-1}(\zeta):=2 i \operatorname{Re}\left(\sum_{1 \leq j+k \leq m-1} \frac{1}{j ! k !} \partial_{1}^{j} \bar{\partial}_{1}^{k} d(0) \zeta_{1}^{j} \bar{\zeta}_{1}^{k}\right):=\sum_{1 \leq j+k \leq m-1} b_{j k}\left(x_{0}\right) \zeta_{1}^{j} \bar{\zeta}_{1}^{k} .
$$

Then, by virtue of the estimates in (3.5), we may write:

$$
\begin{aligned}
b(\zeta)= & \sum_{1 \leq j+k \leq m-1} b_{j k}\left(x_{0}\right) \zeta_{1}^{j} \bar{\zeta}_{1}^{k}+i \operatorname{Re}\left(\sum_{\beta=2}^{n-1} \sum_{j+k \leq m / 2-1} b_{j, k}^{\beta}\left(x_{0}\right) \zeta_{1}^{j} \bar{\zeta}_{1}^{k} \bar{\zeta}_{\beta}\right) \\
& +\mathcal{O}\left(\left|\zeta_{1}\right|^{m}+\left|\zeta^{\prime}\right|\left|\zeta_{1}\right|^{m / 2}+\left|\zeta^{\prime}\right|^{2}+\left|\zeta_{n}\right|\right),
\end{aligned}
$$

where $\zeta^{\prime}=\left(0, \zeta_{2}, \ldots, \zeta_{n-1}, \zeta_{n}\right)$. For $2 \leq v \leq n-1$, let us write

$$
a_{\nu}(\zeta)=\sum_{1 \leq j+k \leq m / 2-1} a_{j, k}^{\nu} \zeta_{1}^{j} \bar{\zeta}_{1}^{k}+\mathcal{O}\left(\left|\zeta_{1}\right|^{m / 2}+\left|\zeta^{\prime}\right|\right)
$$

where $a_{v}$ 's are coordinate functions of $\partial / \partial \zeta_{v}$ in $L_{1}$. Let $x=\left(x_{1}, \ldots, x_{2 n-1}, t\right)$ be the real coordinates of $\zeta$ and set $D_{k}=\partial / \partial x_{k}, 1 \leq k \leq 2 n-1$.

Note that $-i b_{m-1}(\zeta)$ is a smooth real valued function which is an $(m-1)$-th order polynomial in $\zeta_{1}$ and $\bar{\zeta}_{1}$. We let $a(\zeta)$ be a real valued function defined by

$$
a(\zeta):=\frac{\partial}{\partial x_{1}}\left(-i b_{m-1}\right)=\operatorname{Im}\left[\frac{\partial}{\partial \zeta_{1}} \bar{b}_{m-1}\right]:=\sum_{0 \leq j+k \leq m-2} a_{j, k} \zeta_{1}^{j} \bar{\zeta}_{1}^{k}
$$

Using the coefficient functions $b_{j, k}^{\beta}, a_{j, k}^{\nu}$ and $a_{j, k}$ defined in (3.6)-(3.8), we set

$$
\begin{aligned}
& A_{l}\left(x_{0}\right)=\max \left\{\left|a_{j, k}\right| ; j+k=l\right\}, \quad l=0,1,2, \ldots, m-2, \\
& E_{l^{\prime}}\left(x_{0}\right)=\max \left\{\left|a_{j, k}^{v}\right|,\left|b_{j, k}^{\beta}\right| ; j+k=l^{\prime}, \beta, v=2, \ldots, n-1\right\}, \quad 0 \leq l^{\prime} \leq m / 2-1,
\end{aligned}
$$

and for each $\delta>0$ we define

$$
\tau\left(x_{0}, \delta\right)=\min _{\substack{0 \leq l \leq m-2 \\ 0 \leq l^{\prime} \leq m / 2-1}}\left\{\left(\delta / A_{l}\left(x_{0}\right)\right)^{1 /(l+2)},\left(\delta^{1 / 2} / E_{l^{\prime}}\left(x_{0}\right)\right)^{1 /\left(l^{\prime}+1\right)}\right\} .
$$

Set $\tau\left(x_{0}, \delta\right)=\tau$ for a convenience. Then it follows from (3.9) that

$$
\left|\partial_{1}^{j} \bar{\partial}_{1}^{k} a(0)\right| \leq \delta \tau^{-(j+k+2)}, \quad j+k \leq m-2,
$$

and for $2 \leq \beta, v \leq n-1$, we have

$$
\begin{aligned}
& \left|\partial_{1}^{j} \bar{\partial}_{1}^{k} a_{v}(0)\right| \leq \delta^{1 / 2} \tau^{-(j+k+1)}, \\
& \left|\bar{\partial}_{\beta} \partial_{1}^{j} \bar{\partial}_{1}^{k} b(0)\right| \leq \delta^{1 / 2} \tau^{-(j+k+1)}, \quad j+k \leq m / 2-1 .
\end{aligned}
$$


If we combine the estimates in (3.10) with the fact that $D_{2}^{s} b_{m-1}(0)=0, s \leq m$ (because $\left.\left(\partial_{1}-\bar{\partial}_{1}\right)^{s} d(0)=0, s \leq m\right)$, we obtain, from the expression of $a(\zeta)$ in (3.8), that

$$
\left|\tilde{\partial}_{\beta}^{i} \partial_{1}^{j} \bar{\partial}_{1}^{k} b(0)\right| \leq \delta^{1-i / 2} \tau^{-(j+k+1)}, \quad i=0,1, \quad i \cdot m / 2+j+k \leq m-1 .
$$

From the estimates in (3.10)-(3.13) together with the fact that $\eta\left(L_{1}\right) \equiv 0$ it follows, by induction, that

$$
\left|\partial_{1}^{j} \bar{\partial}_{1}^{k} \eta\left(\frac{\partial}{\partial \zeta_{1}}\right)(0)\right| \lesssim \delta^{1 / 2} \tau^{-(j+k)}, \quad j+k \leq m / 2-1,
$$

because $\eta\left(\partial / \partial \zeta_{\alpha}\right)(0)=0,1 \leq \alpha \leq n-1$, and $\delta^{1 / 2} \lesssim \tau$.

Set $\omega^{n}=1 / 2(d t+i \eta)$. Since $\mathcal{S}$ is integrable it follows that $\bar{\omega}^{n}\left(\left[L_{1}, L_{\alpha}\right]\right)=0$ along $M$ and hence we have

$$
\partial_{1}^{j} \bar{\partial}_{1}^{k} \eta\left(\left[L_{1}, L_{\alpha}\right]\right)(0)=0, \quad j+k \leq m .
$$

Combining the estimates in (3.5), (3.10)-(3.15) with the fact that $\eta\left(\left[L_{1}, \bar{L}_{\alpha}\right]\right)(0)=0,2 \leq$ $\alpha \leq n-1$, one obtains that

$$
\left|\partial_{1}^{j} \bar{\partial}_{1}^{k} d_{\alpha}(0)\right|,\left|\partial_{1}^{j} \bar{\partial}_{1}^{k} e_{\alpha}(0)\right| \lesssim \delta^{1 / 2} \tau^{-1}, \quad j+k \leq 1,
$$

and hence that

$$
\left|\partial_{1}^{j} \bar{\partial}_{1}^{k} \eta\left(\frac{\partial}{\partial \zeta_{\alpha}}\right)(0)\right| \lesssim \delta^{1 / 2} \tau^{-1}, \quad j+k \leq 1 .
$$

If we use again the estimates in (3.5), (3.10)-(3.15) together with the fact that $\eta\left(L_{\alpha}\right) \equiv 0$, $1 \leq \alpha \leq n-1$, we obtain, by induction, that

$$
\begin{aligned}
& \left|\partial_{1}^{j} \bar{\partial}_{1}^{k} \eta\left(\frac{\partial}{\partial \zeta_{\alpha}}\right)(0)\right| \lesssim \delta^{1 / 2} \tau^{-(j+k)}, \quad j \geq 1, \quad j+k \leq m / 2-1, \\
& \left|\partial_{1}^{j} \bar{\partial}_{1}^{k} d_{\alpha}(0)\right|,\left|\partial_{1}^{j} \bar{\partial}_{1}^{k} e_{\alpha}(0)\right| \lesssim \delta^{1 / 2} \tau^{-(j+k)}, \quad j \geq 1, \quad j+k \leq m / 2-1
\end{aligned}
$$

for $2 \leq \alpha \leq n-1$. To obtain the estimates for the derivatives of the form $\bar{\partial}_{1}^{l}$ for $l \geq 2$, we note, from the estimates in (3.5), that

$$
\left(\bar{\partial}_{1}-\partial\right)^{s} d_{\alpha}(0)=\left(\bar{\partial}_{1}-\partial\right)^{s} e_{\alpha}(0)=0, \quad s \leq m, \quad 2 \leq \alpha \leq n-1 .
$$

Since we can write

$$
\left(\bar{\partial}_{1}-\partial_{1}\right)^{s}=\bar{\partial}_{1}^{s}+\sum_{j=1}^{s-1} c_{j, s} \bar{\partial}_{1}^{s-j} \partial_{1}^{j},
$$

where $c_{j, s}$ 's are integers, we conclude, from the estimates in (3.16)-(3.18), that

$$
\begin{aligned}
& \left|\partial_{1}^{j} \bar{\partial}_{1}^{k} \eta\left(\frac{\partial}{\partial \zeta_{\alpha}}\right)(0)\right| \lesssim \delta^{1 / 2} \tau^{-(j+k)}, \quad j+k \leq m / 2-1, \\
& \left|\partial_{1}^{j} \bar{\partial}_{1}^{k} d_{\alpha}(0)\right|,\left|\partial_{1}^{j} \bar{\partial}_{1}^{k} e_{\alpha}(0)\right| \lesssim \delta^{1 / 2} \tau^{-(j+k)}, \quad j+k \leq m / 2-1
\end{aligned}
$$


for $2 \leq \alpha \leq n-1$. By virtue of the estimates in (3.11)-(3.13) together with (3.19) and (3.20), we obtain by induction that

$$
\left|\tilde{\partial}_{\beta}^{i} \partial_{1}^{j} \bar{\partial}_{1}^{k} \eta\left(\frac{\partial}{\partial \zeta_{1}}\right)(0)\right| \lesssim \delta^{1-i / 2} \tau^{-(j+k+1)}, \quad i=0,1, \quad i \cdot m / 2+j+k \leq m-1
$$

These estimates are essential ingredients to obtain a uniform subelliptic estimates for $\bar{\partial}$ in dilated coordinates.

Set $\tau\left(x_{0}, \delta\right)=\tau$ for convenience and let

$$
R_{\delta}\left(x_{0}\right)=\left\{\zeta \in C^{n} ;\left|\zeta_{1}\right| \leq \tau,\left|\zeta_{\beta}\right| \leq \delta^{1 / 2} \tau^{-1 / 2}, \beta=2, \ldots, n-1,\left|\zeta_{n}\right| \leq \delta\right\},
$$

and define a dilation map $D_{\delta}: \boldsymbol{C}^{n} \rightarrow \boldsymbol{C}^{n}$ by

$$
D_{\delta}(\zeta)=\left(\tau^{-1} \zeta_{1}, \delta^{-1 / 2} \zeta_{2}, \ldots, \delta^{-1 / 2} \zeta_{n-1}, \delta^{-1} \zeta_{n}\right)=:\left(w_{1}, \ldots, w_{n}\right)=w
$$

Define

$Q_{\delta}\left(x_{0}\right)=D_{\delta}\left(R_{\delta}\left(x_{0}\right)\right)=\left\{w \in C^{n} ;\left|w_{1}\right| \leq 1,\left|w_{\beta}\right| \leq \tau^{-1 / 2}, \beta=2, \ldots, n-1,\left|w_{n}\right| \leq 1\right\}$ and set $L_{1}^{\delta}=\tau\left(D_{\delta}\right)_{*} L_{1}$. Then, from the expression of $L_{1}$ in (3.4), we have

$$
\begin{aligned}
L_{1}^{\delta}= & \frac{\partial}{\partial w_{1}}+a_{1}\left(D_{\delta}(w)\right) \frac{\partial}{\partial w_{1}}+b_{1}\left(D_{\delta}(w)\right) \frac{\partial}{\partial \bar{w}_{1}}+\sum_{l=2}^{n-1} \tau \delta^{-1 / 2} a_{l}\left(D_{\delta}^{-1}(w)\right) \frac{\partial}{\partial w_{l}} \\
& +\sum_{l=2}^{n-1} \tau \delta^{-1 / 2} b_{l}\left(D_{\delta}^{-1}(w)\right) \frac{\partial}{\partial \bar{w}_{l}}+\tau \delta^{-1} b\left(D_{\delta}^{-1}(w)\right) \frac{\partial}{\partial y_{2 n-1}}
\end{aligned}
$$

where $w_{n}=y_{2 n}+i y_{2 n-1}$. Set $\eta_{\delta}=\delta^{-1} \eta$. Therefore, $\eta_{\delta}\left(\partial / \partial y_{2 n-1}\right)=1$ on $M \cap U$.

Set $B(w)=b\left(D_{\delta}^{-1}(w)\right)$. Recall the expression of $B(w)$ defined in (3.6). So we can write:

$$
\begin{aligned}
\tau \delta^{-1} B(w)= & \sum_{1 \leq j+k \leq m-1} b_{j k}\left(x_{0}\right) \delta^{-1} \tau^{j+k+1} w_{1} \bar{w}_{1}^{k} \\
& +i \operatorname{Re}\left(\sum_{\beta=2}^{n-1} \sum_{j+k \leq m / 2-1} b_{j, k}^{\beta}\left(x_{0}\right) \delta^{-1 / 2} \tau^{j+k+1} w_{1} \bar{w}_{1} \bar{w}_{\beta}\right)+\mathcal{O}(\tau) .
\end{aligned}
$$

Then as in Section 2 of [7], the non-negative condition for $i / 2 \eta_{\delta}\left(\left[L_{1}^{\delta}, \bar{L}_{1}^{\delta}\right]\right)$ on $Q_{\delta}\left(x_{0}\right) \cap M$ forces that

$$
\left|\delta^{-1 / 2} \tau^{j+k+1} b_{j, k}^{\beta}\left(x_{0}\right)\right| \lesssim \tau^{\gamma} \ll 1, \quad j+k \leq \frac{m}{2}-1,
$$

where $\gamma=(10 \times(m / 2) !)^{-1}$. Therefore the $b_{j, k}^{\beta}$ 's, in the Taylor expansion of $b(\zeta)$, are not the major terms in the definition of $\tau\left(x_{0}, \delta\right)$ in (3.9), and the estimates in (3.22) show that

$$
\left|\bar{\partial}_{\beta} \partial_{1}^{j} \bar{\partial}_{1}^{k} b(0)\right| \lesssim \delta^{1 / 2} \tau^{-(j+k+1)+\gamma}, \quad j+k \leq m / 2-1, \quad 2 \leq \beta \leq n-1 .
$$


By using the estimates in (3.5), (3.14), (3.15), (3.22) and (3.23) together with the fact that $\eta\left(L_{v}\right) \equiv 0,1 \leq v \leq n-1$, we can also show, by the method leading to (3.19) and (3.20), that

$$
\begin{aligned}
& \left|\partial_{1}^{j} \bar{\partial}_{1}^{k} \eta\left(\frac{\partial}{\partial \zeta_{\alpha}}\right)(0)\right| \lesssim \delta^{1 / 2} \tau^{-j-k+\gamma}, \\
& \left|\partial_{1}^{j} \bar{\partial}_{1}^{k} d_{\alpha}(0)\right|,\left|\partial_{1} \bar{\partial}_{1}^{k} e_{\alpha}(0)\right| \lesssim \delta^{1 / 2} \tau^{-j-k+\gamma}, \quad j+k \leq m / 2-1 .
\end{aligned}
$$

Using the estimates in (3.5) and (3.23)-(3.25), we can also show that

(3.26) $\left|\partial_{1}^{j} \bar{\partial}_{1}^{k} \eta\left(\left[L_{1}, \bar{L}_{\alpha}\right]\right)(0)\right| \lesssim \delta^{1 / 2} \tau^{-(j+k+1)+\gamma}, \quad j+k \leq m / 2-1, \quad 2 \leq \alpha \leq n-1$.

Now we want to show that the coefficients $a_{\nu}(\zeta)$ of $L_{1}$ satisfy the estimates similar to those in (3.26). Recalling the expression of $L_{1}$ in $\zeta$ coordinates as in (3.4), we set

$$
\tilde{L}_{1}=L_{1}-\sum_{\nu=2}^{n-1} a_{\nu}(\zeta) L_{v}, \quad \tilde{L}_{\alpha}=L_{\alpha}, \quad 2 \leq \alpha \leq n-1
$$

Then $\tilde{L}_{1}$ can be written as:

$$
\tilde{L}_{1}=\frac{\partial}{\partial \zeta_{1}}+\sum_{l=1}^{n-1} \tilde{a}_{l}(\zeta) \frac{\partial}{\partial \zeta_{l}}+\sum_{l=1}^{n-1} \tilde{b}_{l}(\zeta) \frac{\partial}{\partial \bar{\zeta}_{l}}+\tilde{b}(\zeta) \frac{\partial}{\partial x_{2 n-1}}
$$

where, from the estimates in (3.5), (3.11) and (3.23)-(3.26), we have

$$
\begin{aligned}
& \partial_{1}^{j} \bar{\partial}_{1}^{k} \tilde{a}_{l}(0)=\partial_{1}^{j} \bar{\partial}_{1}^{k} \tilde{b}_{l}(0)=0, \quad j+k \leq m-1, \quad 2 \leq l \leq n-1, \\
& \left|\partial_{1}^{j} \bar{\partial}_{1}^{k} \tilde{b}(0)\right| \lesssim \delta \tau^{-(j+k+1)}, \quad j+k \leq m-1, \\
& \left|\tilde{\partial}_{\beta} \partial_{1}^{j} \bar{\partial}_{1}^{k} \tilde{b}(0)\right| \lesssim \delta^{1 / 2} \tau^{-(j+k+1)+\gamma}, \quad j+k \leq m / 2-1
\end{aligned}
$$

for $2 \leq \beta \leq n-1$.

If we combine the estimates in (3.27)-(3.29) and apply the methods leading to the estimates in (3.26), we obtain that

(3.30) $\left|\partial_{1}^{j} \bar{\partial}_{1}^{k} \eta\left(\left[\tilde{L}_{1}, \overline{\tilde{L}}_{\alpha}\right]\right)(0)\right| \lesssim \delta^{1 / 2} \tau^{-(j+k+1)+\gamma}, \quad j+k \leq m / 2-1, \quad 2 \leq \alpha \leq n-1$.

Note that

$$
\frac{i}{2} \eta\left(\left[\tilde{L}_{1}, \bar{L}_{\alpha}\right]\right)=\frac{i}{2} \eta\left(\left[L_{1}, \bar{L}_{\alpha}\right]\right)-\frac{i}{2} \sum_{\nu=2}^{n-1} a_{\nu}(\zeta) \eta\left(\left[L_{\nu}, \bar{L}_{\alpha}\right]\right)
$$

where

$$
\frac{i}{2} \eta\left(\left[L_{\alpha}, \bar{L}_{\alpha}\right]\right)(0)=c_{\alpha}\left(x_{0}\right) \geq d_{0}>0, \quad 2 \leq \alpha \leq n-1,
$$

for an independent constant $d_{0}>0$. If we use the fact that $i / 2 \eta\left(\left[L_{\alpha}, \bar{L}_{\beta}\right]\right)(0)=0$ for $\alpha \neq \beta$ together with the estimates in (3.11) and (3.26)-(3.30), we obtain that

$$
\left|\partial_{1}^{j} \bar{\partial}_{1}^{k} a_{v}(0)\right| \lesssim \delta^{1 / 2} \tau^{-(j+k+1)+\gamma}, \quad j+k \leq m / 2-1, \quad 2 \leq v \leq n-1 .
$$


Now set

$$
T=L_{1}+\bar{L}_{1}=\frac{\partial}{\partial x_{1}}+\sum_{l=1}^{2 n-2} a_{l}(x) \frac{\partial}{\partial x_{l}}+a_{2 n-1}(x) \frac{\partial}{\partial x_{2 n-1}} .
$$

Then from the estimates of $a_{2 n-1}$ in (3.5), and of $a_{l}, 3 \leq l \leq 2 n-2$, in (3.31), it follows that

$$
\left|\partial_{1}^{j} \bar{\partial}_{1}^{k} a_{l}(0)\right| \lesssim \delta^{1 / 2} \tau^{-(j+k+1)+\gamma}, \quad j+k \leq m / 2-1, \quad 3 \leq l \leq 2 n-1 .
$$

From (3.31), we conclude that the functions $a_{v}(\zeta)$ are also not the major terms in the definition of $\tau\left(x_{0}, \delta\right)$ in (3.9). Therefore we conclude from (3.23) and (3.32) that

$$
\tau\left(x_{0}, \delta\right)=\min \left\{\left(\delta / A_{l}\left(x_{0}\right)\right)^{1 / l+2} ; 0 \leq l \leq m-2\right\},
$$

and hence it follows that $\delta^{1 / 2} \lesssim \tau \lesssim \delta^{1 / m}$, and if $\delta^{\prime}<\delta^{\prime \prime}$, then

$$
\left(\delta^{\prime} / \delta^{\prime \prime}\right)^{1 / 2} \tau\left(x_{0}, \delta^{\prime \prime}\right) \lesssim \tau\left(x_{0}, \delta^{\prime}\right) \lesssim\left(\delta^{\prime} / \delta^{\prime \prime}\right)^{1 / m} \tau\left(x_{0}, \delta^{\prime \prime}\right) .
$$

In order to study how $\tau\left(x_{0}, \delta\right)$ depends on $x_{0}$, it is convenient to introduce an analogous quantity $\mu(x, \delta)$ that is defined more intrinsically. Let us cover $\bar{M}$ by a finite number of neighborhoods $U_{v}, v=1, \ldots, N$, in $\Omega$ so that in each $U_{v}$, Proposition 3.1 holds. Let $\left\{\chi_{v}\right\}$ be a partion of unity subordinated to the coordinate neighborhoods $\left\{U_{v}\right\}$ of $\Omega$, and let $m$ be a given positive integer.

For any $j, k \geq 0, j \geq 1$, we define

$$
\mathcal{L}_{j, k}^{v} \eta(x)=\frac{i}{2} L_{1}^{j-1} \bar{L}_{1}^{k} \eta\left(\left[L_{1}, \bar{L}_{1}\right]\right)(x), \quad x \in U_{v},
$$

and set

$$
C_{l}^{\nu}(x)=\sum_{j+k=l}\left|\mathcal{L}_{j+k}^{\nu} \eta(x)\right|^{2}, \quad 1 \leq l \leq m-1,
$$

and

$$
C_{l}(x)=\sum_{\nu=1}^{N} \chi_{\nu} C_{l}^{\nu}(x) .
$$

Set $M=(m+1)$ !, and for each $\delta>0$, define

$$
\mu(x, \delta)=\left(\sum_{l=1}^{m} C_{l}^{M / l+1}(x) \delta^{-2 M / l+1}\right)^{-1 / 2 M} .
$$

Note that $\sum_{l=1}^{m} C_{l}(x)>0$ if the type at $x$ is less than or equal to $m$. Therefore $\mu(x, \delta)$ is defined intrinsically as a smooth function of $\delta>0$ and $x$, for $x$ satisfying $\sum_{l=1}^{m} C_{l}(x)>0$. Let us fix $x_{0} \in M \cap U$ and take the smooth complex valued coordinates $\zeta=\left(\zeta_{1}, \ldots, \zeta_{n}\right)$ defined on $M \cap U$ as in Proposition 3.1, where

$$
\zeta_{j}=\frac{1}{2}\left(x_{2 j-1}+i x_{2 j}\right), \quad 1 \leq j \leq n-1, \quad \zeta_{n}=\frac{1}{2}\left(t+i x_{2 n-1}\right) .
$$

For each $\delta>0$, set $\tau_{1}=\tau_{2}=\tau\left(x_{0}, \delta\right)$ and $\tau_{k}=\delta^{1 / 2}, 3 \leq k \leq 2 n-2$, and define

$$
P_{\delta}\left(x_{0}\right)=\left\{x \in \boldsymbol{R}^{2 n} ;\left|x_{i}\right| \leq \tau_{i}, 1 \leq i \leq 2 n-2,\left|x_{2 n-1}\right| \leq \delta,|t| \leq \delta\right\} .
$$


Without loss of generality we may assume that there is $\nu_{0} \in\{1,2, \ldots, N\}$ such that $x_{0} \in U_{\nu_{0}}$ and $\chi_{\nu_{0}} \geq 1 / N$ on $P_{\delta}\left(x_{0}\right)$. Recall that $L_{1}$ can be written, in $\zeta$ coordinates, as

$$
L_{1}=\frac{\partial}{\partial \zeta_{1}}+\sum_{l=1}^{n-1} a_{l}(\zeta) \frac{\partial}{\partial \zeta_{k}}+\sum_{l=1}^{n-1} b_{l}(\zeta) \frac{\partial}{\partial \bar{\zeta}_{l}}+b(\zeta) \frac{\partial}{\partial x_{2 n-1}}
$$

where, from the estimates in (3.5), (3.23) and (3.31), we have

$$
\begin{aligned}
& \left|\partial_{1}^{j} \bar{\partial}_{1}^{k} a_{l}(0)\right|,\left|\tilde{\partial}_{\beta} \partial_{1}^{j} \bar{\partial}_{1}^{k} b(0)\right| \lesssim \delta^{1 / 2} \tau^{-(j+k+1)+\gamma}, \quad j+k \leq m / 2-1, \\
& \left|\partial_{1}^{j} \bar{\partial}_{1}^{k} b(0)\right| \lesssim \delta \tau^{-(j+k+1)}, \quad j+k \leq m-1, \\
& \partial_{1}^{j} \bar{\partial}_{1}^{k} b_{l}(0)=0, \quad j+k \leq m, \quad 2 \leq l, \beta \leq n-1 .
\end{aligned}
$$

Therefore we may write

$$
\mathcal{L}_{j, k} \eta(\zeta)=-\partial_{1}^{j-1} \bar{\partial}_{1}^{k}\left[\operatorname{Im}\left(\frac{\partial}{\partial \zeta_{1}} \bar{b}(\zeta)\right) \eta\left(\frac{\partial}{\partial x_{2 n-1}}\right)\right]+E_{j+k},
$$

where $E_{j, k}$ satisfies, from the estimates in (3.37), that

$$
\left|\tilde{\partial}_{\beta}^{i} \partial_{1}^{l_{1}} \bar{\partial}_{1}^{l_{2}} E_{j+k}(0)\right| \lesssim \delta^{1-i / 2} \tau^{-\left(j+k+l_{1}+l_{2}+1\right)+\gamma}
$$

for $i=0,1$, and $i \cdot m / 2+j+k+l_{1}+l_{2} \leq m-1$. From (3.37)-(3.39) it follows that

$$
\left|\tilde{\partial}_{\beta}^{i} \partial_{1}^{l_{1}} \bar{\partial}_{1}^{l_{2}} \mathcal{L}_{j, k} \eta(0)\right| \lesssim \delta^{1-i / 2} \tau^{-\left(j+k+l_{1}+l_{2}+1\right)}
$$

for $i=0,1$, and $i \cdot m / 2+j+k+l_{1}+l_{2} \leq m-1$. By (3.39), (3.40) combined with a simple Taylor's theorem argument, we then have

$$
\left|\mathcal{L}_{j, k} \eta(\zeta)\right| \lesssim \delta \tau^{-(j+k+1)}, \quad \zeta \in P_{\delta}\left(x_{0}\right)
$$

By virtue of the definition of $\mu(x, \delta)$ in (3.35), (3.41) implies that

$$
\mu(x, \delta) \gtrsim \tau\left(x_{0}, \delta\right), \quad x \in P_{\delta}\left(x_{0}\right) .
$$

Conversely, let us show that $\mu(x, \delta) \lesssim \tau\left(x_{0}, \delta\right)$ for $x \in P_{\delta}\left(x_{0}\right)$. Recall that $\tau\left(x_{0}, \delta\right)$ is actually defined as in (3.33). Set

$$
T\left(x_{0}, \delta\right)=\min \left\{l ;\left(\delta / A_{l}\left(x_{0}\right)\right)^{1 / l+2}=\tau\left(x_{0}, \delta\right)\right\} .
$$

Therefore there must exist integers $j_{0}, k_{0}$ with $\left(j_{0}-1\right)+k_{0}=T\left(x_{0}, \delta\right)$ such that

$$
\begin{aligned}
\left|\frac{1}{\left(j_{0}-1\right) ! k_{0} !} \partial_{1}^{j_{0}-1} \bar{\partial}_{1}^{k_{0}} a(0)\right| & =\left|\frac{1}{\left(j_{0}-1\right) ! k_{0} !} \partial_{1}^{j_{0}-1} \bar{\partial}_{1}^{k_{0}}\left[\operatorname{Im} \frac{\partial}{\partial \zeta_{1}} \bar{b}\right](0)\right| \\
& =\delta \tau^{-j_{0}-k_{0}-1} .
\end{aligned}
$$

Assuming that $\chi_{\nu_{0}} \geq 1 / N$, we obtain from the estimates in (3.38), (3.39) and (3.44) that

$$
\left|\mathcal{L}_{j_{0}, k_{0}}^{\nu_{0}} \eta\left(x_{0}\right)\right| \geq \frac{1}{2 N}\left(j_{0}-1\right) ! k ! \delta \tau\left(x_{0}, \delta\right)^{-j_{0}-k_{0}-1},
$$

provided that $\delta$ is sufficiently small. Again, by using the estimates in (3.38)-(3.44) and the Taylor series method, we obtain that

$$
\left|\mathcal{L}_{j_{0}, k_{0}}^{\nu_{0}} \eta(x)\right| \approx \delta \tau\left(x_{0}, \delta\right)^{-j_{0}-k_{0}-1}, \quad x \in P_{\delta}\left(x_{0}\right) .
$$


If we combine (3.45) and the definition of $\mu(x, \delta)$, we obtain that

$$
\mu(x, \delta) \lesssim \tau\left(x_{0}, \delta\right), \quad x \in P_{\delta}\left(x_{0}\right) .
$$

Combining (3.42) and (3.46), we have proved the following proposition.

PROPOSITION 3.2. If $x \in P_{\delta}\left(x_{0}\right)$, then

$$
\tau\left(x_{0}, \delta\right) \approx \mu(x, \delta) .
$$

Corollary 3.3. Suppose $x \in P_{\delta}\left(x_{0}\right)$. Then

$$
\tau\left(x_{0}, \delta\right) \approx \tau(x, \delta) .
$$

PROOF. If we set $x=x_{0}$ in (3.47), we see that $\mu(x, \delta) \approx \tau\left(x_{0}, \delta\right)$. Since this holds for $x_{0}=x$, it follows that $\mu(x, \delta) \approx \tau(x, \delta)$. Hence (3.48) follows.

REMARK 3.4. $\mu(x, \delta)$ is defined intrinsically. That is, it does not depend on the choice of a specific coordinates. Propositions 3.2 and Corollary 3.3 show that the quantity $\tau(x, \delta)$ is also defined invariantly, up to a universal constant, with respect to the coordinate functions.

Now we want to construct a family of plurisubharmonic functions with large Hessian. The existence of these functions will be a crucial ingredient in the subelliptic estimates for $\bar{\partial}$-type equation. Note that we are free to choose $x_{0} \in M$ and $\delta>0$. Now assume that $x^{\nu} \in M$. Let us take the special coordinates $\zeta=\left(\zeta_{1}, \ldots \zeta_{n}\right)$ defined near $x^{\nu}$ and write the vector fields $L_{1}, \ldots, L_{n}$ as in (3.4) satisfying (3.5). Also, let $T\left(x_{0}, \delta\right)$ be defined in (3.43). Let $x=\left(x^{\prime}, t\right)$ be the real coordinates for $\zeta$, where $\left(x^{\nu}, 0\right)=x^{\nu} \in M$. Set $\tau_{1}=\tau\left(x^{\nu}, \delta\right)$, $\tau_{\beta}=\delta^{1 / 2}, 2 \leq \beta \leq n-1$, and $\tau_{n}=\delta$, and put $\alpha=\left(\alpha_{1}, \ldots \alpha_{n}\right)$.

Proposition 3.5. Suppose $x^{\nu} \in M \cap U$. Then there exist a small constant $a>0$ and a smooth function $h_{x^{\nu}, \delta}$ on $M \cap U$ which satisfy the following:

(i) $\left|h_{x^{v}, \delta}(x)\right| \leq 1$ and $h_{x^{v}, \delta} \in C_{0}^{\infty}\left(P_{\delta}\left(x^{\nu}\right)\right)$.

(ii) If $|t| \leq a \delta$ and if $h_{x^{\nu}, \delta}$ is not plurisubharmonic at $x=\left(x^{\prime}, t\right)$, then

$$
T\left(x^{\prime}, a \delta\right)<T\left(x^{\nu}, \delta\right) .
$$

(iii) If $x \in P_{a \delta}\left(x^{\nu}\right),|t| \leq a \delta$, and if the inequality

$$
\partial \bar{\partial} h_{x^{\nu}, \delta}(x)(L, \bar{L}) \gtrsim \prod_{k=1}^{n} \tau_{k}^{-2}\left|b_{k}\right|^{2}
$$

fails to hold at $x=\left(x^{\prime}, t\right)$ for $L=\sum_{j=1}^{n} b_{j} L_{j}$, then $T\left(x^{\prime}, a \delta\right)<T\left(x^{v}, \delta\right)$.

(iv) For all $x \in P_{\delta}\left(x^{\nu}\right)$ and all $L=\sum_{j=1}^{n} b_{j} L_{j}$ at $x$,

$$
\left|\partial \bar{\partial} h_{x^{v}, \delta}(L, \bar{L})\right| \lesssim \prod_{k=1}^{n} \tau_{k}^{-2}\left|b_{k}\right|^{2} .
$$

(v) $\left|D^{\alpha} h_{x^{\nu}, \delta}(x)\right| \leq C_{\alpha} \prod_{k=1}^{n} \tau_{k}^{-\alpha_{k}}$, where $D^{\alpha}=\partial_{1}^{\beta_{1}} \bar{\partial}_{1}^{\gamma_{1}} \cdots \partial_{n}^{\beta_{n}} \bar{\partial}_{n}^{\gamma_{n}}, \alpha_{i}=\beta_{i}+\gamma_{i}$. 
Proof. We use the definitions of $\mu(x, \delta), \tau(x, \delta), T(x, \delta)$ and the fact that $\tau\left(x^{v}, \delta\right) \approx$ $\mu(x, \delta)$ for $x \in P_{\delta}\left(x^{\nu}\right)$. Then the proof of (i) through (v) follow the same lines as in the proof of Proposition 3.1 in [7].

For each $\varepsilon>0$, we set $\Omega_{\varepsilon}=M \times(-1, \varepsilon)$ and $S(\varepsilon)=M \times(-\varepsilon, \varepsilon)$. By adding up the functions $h_{x^{\nu}, \delta}$ constructed in Proposition 3.5, we can construct bounded plurisubharmonic weight functions so that the Hessian of these functions satisfy certain essentially maximal bounds in a thin strip $S(\varepsilon)$ of $M_{0}$. The heart of these construction is the so-called "doubling property" of $P_{\delta}\left(x_{0}\right)$, which comes from the relation in (3.48). For a detailed proof of the following theorem, one can refer to Section 3 of [4]. For each small $\delta>0$, we set $\tau_{1}(x)=$ $\tau(x, \delta), \tau_{2}(x)=\cdots \tau_{n-1}(x)=\delta^{1 / 2}$ and $\tau_{n}(x)=\delta$ as before.

THEOREM 3.6. For all small $\delta>0$, there is a plurisubharmonic function $\psi_{\delta} \in$ $C^{\infty}\left(\Omega_{\delta}\right)$ which satisfies the following.

(i) $\left|\psi_{\delta}(x)\right| \leq 1, x \in U \cap \Omega_{\delta}$.

(ii) For all $L=\sum_{j=1}^{n} b_{j} L_{j}$ at $x \in U \cap S(\delta)$,

$$
\partial \bar{\partial} \psi_{\delta}(x)(L, \bar{L}) \approx \sum_{j=1}^{n}\left|b_{j}(x)\right|^{2} \tau_{j}^{-2}(x), \quad \text { and }
$$

(iii) $\left|D^{\alpha} \psi_{\delta}(x)\right| \lesssim C_{\alpha} \prod_{k=1}^{n} \tau_{k}^{-\alpha_{k}}(x)$, where $D^{\alpha}=\partial_{1}^{\beta_{1}} \bar{\partial}_{1}^{\gamma_{1}} \cdots \partial_{n}^{\beta_{n}} \bar{\partial}_{n}^{\gamma_{n}}, \alpha_{i}=\beta_{i}+\gamma_{i}$.

In $D_{2}$-equation, we will assign a Dirichlet condition on one side of $b S_{g}^{+}$, and the Neumann condition on the other side of $b S_{g}^{+}$. This fact leads us to another difficulty which was not occurred in 1/2-subelliptic estimates of Catlin in [5]. To overcome this difficulty, we need the following Lemma 3.7 and Proposition 3.8, which will be used in the proof of subelliptic estimates for $D_{2}$-equation in Section 5 .

LEMMA 3.7. Let $x^{\nu} \in M \cap U$ and $\operatorname{set} c_{1 k}^{n}=\omega^{n}\left(\left[L_{1}, \bar{L}_{k}\right]\right), 1 \leq k \leq n$. Then for each small $\delta>0$, we have

$$
\begin{aligned}
& \left|c_{11}^{n}(x)\right| \lesssim \delta \tau\left(x^{\nu}, \delta\right)^{-2}, \quad x \in P_{\delta}\left(x^{\nu}\right), \\
& \left|c_{1 k}^{n}(x)\right| \lesssim \delta^{1 / 2} \tau\left(x^{\nu}, \delta\right)^{-1+\gamma}, \quad x \in P_{\delta}\left(x^{\nu}\right), \quad 2 \leq k \leq n,
\end{aligned}
$$

where $\gamma=(10 \times(\mathrm{m} / 2) !)^{-1}$.

Proof. Along $M \cap U$, we have $d t=1 / 2(d t+i \eta)+1 / 2(d t-i \eta)$ and $L_{n}=\partial / \partial t-$ $i \partial / \partial x_{2 n-1}$, which imply that $\partial t=1 / 2(d t+i \eta)$. Hence (3.50) follows from (3.41). Since $\mathcal{L}^{0}$ is integrable to infinite order along $M_{0}$, it follows that

$$
\partial \bar{\partial} t\left(L_{1}, \bar{L}_{n}\right)=\partial \bar{\partial} t\left(L_{1}+\bar{L}_{1}, \bar{L}_{n}\right)=\frac{i}{2} \eta\left(\left[L_{1}+\bar{L}_{1}, \frac{\partial}{\partial t}+i \frac{\partial}{\partial x_{2 n-1}}\right]\right)
$$

along $M \cap U$. Note that we can write $L_{1}+\bar{L}_{1}=T=T_{1}+t T_{2}+x_{2 n-1} T_{3}$, where the coefficient functions of $T_{1}$ does not depend on $t$ or $x_{2 n-1}$. From (3.5) together with the estimates of the 
coefficient functions in (3.37) it follows that

$$
\begin{aligned}
& \left|\partial_{1}^{j} \bar{\partial}_{1}^{k} \frac{\partial}{\partial t} \eta\left(T_{1}\right)(0)\right|, \quad\left|\partial_{1}^{j} \bar{\partial}_{1}^{k} \frac{\partial}{\partial x_{2 n-1}} \eta\left(T_{1}\right)(0)\right| \lesssim \delta^{1 / 2} \tau^{-(j+k+1)+\gamma}, \\
& j+k \leq m / 2-1 .
\end{aligned}
$$

Since $\eta\left(L_{1}+\bar{L}_{1}\right)=\eta(T) \equiv 0$, we have

$$
\partial_{1}^{j} \bar{\partial}_{1}^{k} \frac{\partial}{\partial t} \eta(T)(0)=\partial_{1}^{j} \bar{\partial}_{1}^{k} \frac{\partial}{\partial x_{2 n-1}} \eta(T)(0)=0, \quad j+k \leq m-1 .
$$

Combining (3.53) and (3.54), we obtain that

$$
\left|\partial_{1}^{j} \bar{\partial}_{1}^{k} \eta\left(T_{2}\right)(0)\right|,\left|\partial_{1}^{j} \bar{\partial}_{1}^{k} \eta\left(T_{3}\right)(0)\right| \lesssim \delta^{1 / 2} \tau^{-(j+k+1)+\gamma}, \quad j+k \leq m / 2-1 .
$$

Therefore it folows that

$$
\left|\partial_{1}^{j} \bar{\partial}_{1}^{k} \eta\left(\left[T, \frac{\partial}{\partial t}+i \frac{\partial}{\partial x_{2 n-1}}\right]\right)(0)\right| \lesssim \delta^{1 / 2} \tau^{-(j+k+1)+\gamma}, \quad j+k \leq m / 2-1,
$$

and this proves (3.51) for $k=n$.

When $2 \leq k \leq n-1$, we use the estimates in (3.26).

For each $\delta>0$, let $\psi_{\delta}$ be the function constructed in Theorem 3.6. We need the following proposition which will be used to prove Lemma 5.6 that is necessary for the estimates of (5.39) and (5.40) in the subelliptic estimates of $D_{2}$ operator in Section 5.

PROPOSITION 3.8. For all small $\delta>0$ and for each $\alpha=\left(\alpha_{1}, \ldots \alpha_{n}\right)$ we have

$$
\begin{aligned}
& \left|c_{11}^{n}(x) D^{\alpha} \psi_{\delta}(x)\right| \leq C_{\alpha} \delta \tau(x, \delta)^{-2} \prod_{k=1}^{n} \tau_{k}(x, \delta)^{-\alpha_{k}}, \\
& \left|c_{1, l}^{n}(x) D^{\alpha} \psi_{\delta}(x)\right| \leq C_{\alpha} \delta^{1 / 2} \tau(x, \delta)^{-1+\gamma} \prod_{k=1}^{n} \tau_{k}(x, \delta)^{-\alpha_{k}}
\end{aligned}
$$

for $x \in S(\delta) \cap U$. Here $\tau_{1}(x, \delta)=\tau(x, \delta), \tau_{k}(x, \delta)=\delta^{1 / 2}, 2 \leq k \leq n-1$ and $\tau_{n}(x, \delta)=\delta$.

PROOF. Note that the functions $\psi_{\delta}$ in Theorem 3.6 were constructed by adding up functions $h_{x^{v}, \delta}, x^{v} \in M \cap U$, constructed in Proposition 3.5, with $\operatorname{supp} h_{x^{v}, \delta} \subset P_{\delta}\left(x^{v}\right)$. By virtue of (3.48), there is a small $c>0$, independent of $\delta>0$, so that we can arrange points $x^{\nu}=x^{\nu}(\delta) \in M \cap U, v \in I$, satisfying

$$
S(\delta) \cap U \subset \cup_{\nu \in I} P_{\delta}\left(x^{\nu}\right), \quad \text { and } \quad P_{c \delta}\left(x^{\nu}\right) \cap P_{c \delta}\left(x^{\mu}\right)=\emptyset \quad \text { if } \quad \nu \neq \mu .
$$

Then, as in the proof of Lemma 3.3 in [4], there is a fixed integer $N$ (independent of $\delta$ ) such that any $(N+1)$ intersection of $P_{\delta}\left(x^{v}\right)$ 's are empty.

Now assume that $x \in S(\delta) \cap U$. Then there are $v_{1}, \ldots, v_{l} \in I, l \leq N$, such that $x \in P_{\delta}\left(x^{v_{j}}\right), 1 \leq j \leq l$. By virtue of (3.48) again, it follows that

$$
\tau(x, \delta) \approx \tau\left(x^{\nu_{1}}, \delta\right) \approx \cdots \approx \tau\left(x^{\nu_{l}}, \delta\right),
$$


independent of $\delta$. If we express the vector fields $L_{1}, \ldots, L_{n}$ in terms of the special coordinates in each neighborhood of $x^{v_{i}}, i=1, \ldots, l$, then (3.54) and (3.55) follow from (3.50) and (3.51), respectively.

4. Special frames for almost complex structures. Assume $\bar{M} \subset \tilde{M}$ and let $\varphi \in$ $C^{\infty}(\bar{M})$ be a smooth real-valued function such that $\varphi(x)>0$ for $x \in M$, and $\varphi(x)=0$, $d \varphi(x) \neq 0$ for $x \in b M$. We can extend $\varphi$ to $\Omega$ by requiring that it be independent of $t$. Let us denote by $T_{p}$ the type at a point $p \in \bar{M}$ and define

$$
T(\bar{M})=\max \left\{T_{p} ; p \in \bar{M}\right\} .
$$

Since the type condition is an open condition, it follows that $T(\bar{M})$ is well-defined and is finite. In the sequal, we assume that $T(\bar{M})=m<\infty$. We define $r \in C^{\infty}(\Omega)$ by $r(x, t)=$ $t(\phi(x))^{-2 m}$ and for any $\varepsilon, \sigma, 0<\varepsilon \leq \sigma \leq 1$, define

$$
S_{\varepsilon, \sigma}=\left\{(x, t) \in \Omega ; \varphi(x)>0 \text { and } 0 \leq r(x, t) \leq \varepsilon \sigma^{2 m}\right\} .
$$

REMARK 4.1. The quantities $\varepsilon$ and $\sigma$ will be fixed later. If we set $g(x)=\varepsilon \cdot \sigma^{2 m}$. $\varphi(x)^{2 m}$, then $g$ is the required positive function in the definition of $S_{g}^{+}$in Section 1 and $S_{\varepsilon, \sigma}$ equals to $S_{g}^{+}$.

We define a subbundle of $\mathcal{L}^{0}$ on $S_{\varepsilon, \sigma}$ by letting $\mathcal{R}_{(x, t)}=\left\{L \in \mathcal{L}_{(x, t)}^{0} ; L r=0\right\}$. Clearly, the map $H$ defined by $H(L)=L-(L r)\left(L_{n} r\right)^{-1} L_{n}$ defines an isomorphism of $\mathcal{S}$ onto $\mathcal{R}$ (at all points of $\left.S_{\varepsilon, \sigma}\right)$. Set $\mu_{1}(x)=\mu\left(x, \varepsilon \phi(x)^{2 m}\right), \mu_{2}(x)=\ldots, \mu_{n-1}(x)=\varepsilon^{1 / 2} \phi(x)^{m}$, $\mu_{n}(x)=\varepsilon \phi(x)^{2 m}$. We define a weighted metric $\langle$,$\rangle on \mathcal{L}^{0}$ by the relations:

$$
\begin{aligned}
& \left\langle H\left(L_{j}\right), H\left(L_{k}\right)\right\rangle=\mu_{j}(x)^{-1} \mu_{k}(x)^{-1}\left\langle L_{j}, L_{k}\right\rangle_{0}, \quad 1 \leq j, k \leq n-1 \\
& \left\langle L_{n}, L_{n}\right\rangle=\varepsilon^{-2} \varphi(x)^{-4 m} \\
& \left\langle L_{n}, H\left(L_{l}\right)\right\rangle=0, \quad 1 \leq l \leq n-1,
\end{aligned}
$$

where $L_{l} \in \mathcal{S}, 1 \leq l \leq n-1$. Since $\mu(x, \delta)$ is a smooth function of $x$ and $\delta$, it follows that $\langle$,$\rangle is a smooth Hermitian metric on \mathcal{L}^{0}$. Now, using Proposition 3.1, we shall cover $S_{\varepsilon, \sigma}$ by special (dialated) coordinate neighborhoods such that on each of them, there is a frame $\mathcal{L}$ satisfying required good estimates.

Proposition 4.2. There exist constants $\varepsilon_{0}$ and $\sigma_{0}$ such that if $0<\varepsilon<\varepsilon_{0}$ and $0<$ $\sigma<\sigma_{0}$, then on $S_{\varepsilon, \sigma}$ there exists for all $x_{0} \in M$ with $\varphi\left(x_{0}\right)>0$ a neighborhood $W\left(x_{0}\right) \subset$ $S_{\varepsilon, \sigma}$ with the following properties:

(i) On $W\left(x_{0}\right)$ there are smooth coordinates $y_{1}, \ldots, y_{2 n}$ so that $W\left(x_{0}\right)=\left\{y ;\left|y^{\prime}\right|<\right.$ $\left.\sigma, 0 \leq y_{2 n} \leq \sigma^{2 m}\right\}$, where $y^{\prime}=\left(y_{1}, y_{2}, \ldots, y_{2 n-1}\right)$ is independent of $t$ and the function $y_{2 n}$ is defined by $y_{2 n}=\varepsilon^{-1} \varphi(x)^{-2 m} t$. Thus, $M_{0} \cap W\left(x_{0}\right)$ and $M_{\sigma} \cap W\left(x_{0}\right)$ correspond to the points in $W\left(x_{0}\right)$ where $y_{2 n}=0$ and $\sigma^{2 m}$, respectively. Moreover, the point $\left(x_{0}, 0\right) \in \Omega$, which we identify with $x_{0}$, corresponds to the origin. 
(ii) The above coordinate charts are uniformly smoothly related in the sense that if $W\left(\tilde{p}_{0}\right)$ and $W\left(x_{0}\right)$ intersect, and if $\tilde{y}$ and $y_{0}$ are the associated coordinates, then

$$
\left|D^{\alpha}\left(\tilde{y} \circ\left(y_{0}\right)^{-1}\right)\right| \leq C_{|\alpha|}
$$

holds on that portion of $\boldsymbol{R}^{2 n}$ where $\tilde{y} \circ\left(y_{0}\right)^{-1}$ is defined. The constant $C_{|\alpha|}$ is independent of $\varepsilon, \sigma$, and $x_{0}$,

(iii) On $W\left(x_{0}\right)$, there exists a smooth frame $\left\{L_{1}, \ldots, L_{n}\right\}$ for $\mathcal{L}$ such that if $\left\{\omega^{1}, \ldots, \omega^{n}\right\}$ is the dual frame, and if $L_{k}$ and $\omega^{k}$ are written as $\sum_{j=1}^{2 n} b_{k_{j}} \partial / \partial y_{j}$ and $\sum_{j=1}^{2 n} d_{k j} d y_{j}$, then

$$
\sup _{y \in W\left(x_{0}\right)}\left\{\left|D_{y}^{\alpha} b_{k j}(y)\right|+\left|D_{y}^{\alpha} d_{k j}(y)\right|\right\} \leq C_{|\alpha|},
$$

where $C_{|\alpha|}$ is independent of $x_{0}, j, k, \varepsilon$ and $\sigma$.

(iv) With the frames as in (iii), set $c_{1 l}^{n}=\omega^{n}\left(\left[L_{1}, \bar{L}_{l}\right]\right), l=2, \ldots, n$. Then there is a constant $C>0$ independent of $x_{0}, \varepsilon$ and $\sigma$ such that

$$
\sup _{y \in W\left(x_{0}\right)}\left|c_{1 l}^{n}(y)\right| \leq C \sigma^{2 m} .
$$

(v) There are constants $c>0$ and $C>0$ independent of $x_{0}, \varepsilon$ and $\sigma$ such that if $B_{b}(x)$ denotes the ball of radius $b$ about $x \in S_{\varepsilon, \sigma}$ with respect to the metric $\langle$,$\rangle , then$

$$
B_{c \sigma}\left(x_{0}\right) \subset W\left(x_{0}\right) \subset B_{C \sigma}\left(x_{0}\right)
$$

and if $\operatorname{Vol} B_{b}\left(x_{0}\right)$ denotes the volume of $B_{b}\left(x_{0}\right)$ with respect to $\langle$,$\rangle , then$

$$
c b^{2 n-1} \sigma^{2 m} \leq \operatorname{Vol} B_{b}\left(x_{0}\right) \leq C b^{2 n-1} \sigma^{2 m} .
$$

Proof. We first cover $\bar{M}$ by a finite number of neighborhoods $V_{v}, v=1, \ldots, N$, in $\Omega$ such that in each $V_{v}$ there exist coordinates $\left(u_{1}, \ldots, u_{2 n}\right)$ with the property that $u_{2 n}=t$ and that $u_{k}\left(u^{\prime}, t\right)=u_{k}\left(u^{\prime}, 0\right), k<2 n$, for $\left(u^{\prime}, t\right) \in V_{v}$, and that $\partial / \partial u_{2 n-1}=-X_{0}$ at all points of $M \cap V_{v}$. Also, we can arrange the neighborhoods $V_{v}$ so that Proposition 3.1 holds on each $V_{v}$.

For any point $x_{0} \in M \cap V_{\nu}$, we take coordinate functions $\zeta^{\nu}=\left(\zeta_{1}^{\nu}, \ldots, \zeta_{n}^{\nu}\right)$ constructed as in Proposition 3.1. Let us set $\zeta^{\nu}=\zeta$ and denote by $L_{k}^{\nu}$, the vector fields $L_{k}, 1 \leq k \leq n$, written in $\zeta^{\nu}$-coordinates, and let $x=\left(x_{1}, \ldots, x_{2 n-1}, t\right)$ be the real coordinates of $\zeta$. In $\zeta$-coordinates, we may write:

$$
L_{k}^{v}=\frac{\partial}{\partial \zeta_{k}}+\sum_{l=1}^{n-1} a_{l}^{k}(\zeta) \frac{\partial}{\partial \zeta_{l}}+\sum_{l=1}^{n-1} b_{l}^{k}(\zeta) \frac{\partial}{\partial \bar{\zeta}_{l}}+\left(e_{k}(\zeta)+i d_{k}(\zeta)\right) \frac{\partial}{\partial x_{2 n-1}}, \quad 1 \leq k \leq n,
$$

where the coefficients $a_{l}^{k}, b_{l}^{k}, e_{k}, d_{k}$ satisfy the estimates in (3.5) and (3.37).

Set $\delta=\varepsilon \varphi\left(x_{0}\right)^{2 m}$, and let us write $b(\zeta)=e_{1}(\zeta)+i d_{1}(\zeta)$, where $e_{1}(\zeta)$ and $d_{1}(\zeta)$ are smooth real valued functions. Recall the Taylor expansion of $b(\zeta)$ at $x_{0}$ (i.e., at $\zeta=0$ ) in (3.6). We then take the quantity $\tau\left(x_{0}, \delta\right)$ and the corresponding quantity $\mu(x, \delta)$ defined in (3.9) and (3.35), respectively. By Proposition 3.5, it follows that $\mu\left(x_{0}, \delta\right) \approx \tau\left(x_{0}, \delta\right)$, and 
hence the estimates in (3.37) imply that

$$
\begin{aligned}
& \left|\partial_{1}^{j} \bar{\partial}_{1}^{k} b(0)\right| \lesssim \delta \mu\left(x_{0}, \delta\right)^{-j-k-1}, \quad j+k \leq m-1, \\
& \left|\tilde{\partial}_{\beta} \partial_{1}^{j} \bar{\partial}_{1}^{k} b(0)\right| \lesssim \delta^{1 / 2} \mu\left(x_{0}, \delta\right)^{-(j+k+1)+\gamma}, \quad j+k \leq m / 2-1,
\end{aligned}
$$

where $\tilde{\partial}_{\beta}=\partial_{\beta}$ or $\bar{\partial}_{\beta}, 2 \leq \beta \leq n-1$, and $\partial_{k}=\partial / \partial \zeta_{k}, 1 \leq k \leq n$, and $\gamma=(10 \times(m / 2) !)^{-1}$.

Set $\mu=\mu\left(x_{0}, \delta\right)$ for a convenience. We define new coordinates $y=D_{\varepsilon, x_{0}}(x)=$ $\left(y_{1}, \ldots, y_{2 n}\right)$ by means of dilation map $D_{\varepsilon, x_{0}}: \boldsymbol{R}^{2 n} \rightarrow \boldsymbol{R}^{2 n}$ given by

$$
y=\left(\mu^{-1} x_{1}, \mu^{-1} x_{2}, \delta^{-1 / 2} x_{3}, \ldots, \delta^{-1 / 2} x_{2 n-2}, \delta^{-1} x_{2 n-1}, e^{-1} \varphi(x)^{-2 m} x_{2 n}\right),
$$

where $\varphi(x)$ is the function $\varphi$ expressed in the $x$-coordinates of $x_{0}$. In terms of the $y$-coordinates we define an open set $W_{b}\left(x_{0}\right)$ by

$$
W_{b}\left(x_{0}\right)=\left\{x \in V_{v} \cap S_{\varepsilon, \sigma} ;\left|y_{k}(x)\right|<b, 1 \leq k \leq 2 n-1,0 \leq y_{2 n}(x) \leq \sigma^{2 m}\right\} .
$$

Note that in $W_{b}\left(x_{0}\right), y_{2 n}=0$ and $y_{2 n}=\sigma^{2 m}$ coincide with $r=0$ and $r=\varepsilon \sigma^{2 m}$, respectively, on the boundaries of $S_{\varepsilon, \sigma}$. We define a frame $\left\{L_{1}, \ldots, L_{n}\right\}$ in $W_{b}\left(x_{0}\right)$ by setting

$$
\begin{aligned}
& L_{1}=\mu(x, \delta)\left(L_{1}^{v}-r_{1} L_{n}^{v}\right)=\mu(x, \delta) H\left(L_{1}^{v}\right), \\
& L_{k}=\varepsilon^{1 / 2} \varphi(x)^{m}\left(L_{k}^{v}-r_{k} L_{n}^{v}\right)=\varepsilon^{1 / 2} \varphi(x)^{m} H\left(L_{k}^{v}\right), \quad 2 \leq k \leq n-1, \\
& L_{n}=\varepsilon \varphi(x)^{2 m} L_{n}^{v},
\end{aligned}
$$

where $r_{k}=\left(L_{k}^{v} r\right)\left(L_{n}^{v} r\right)^{-1}$, and $L_{k}^{v}$ is the vector field $L_{k}$ written in $x$-coordinates of $V_{\nu}$. Set

$$
L_{k}^{v}=\sum_{l=1}^{2 n-1} e_{k l}(x) \frac{\partial}{\partial x_{l}}, \quad 1 \leq k \leq n-1, \quad \text { and } \quad L_{n}^{v}=\frac{\partial}{\partial t}+\sum_{l=1}^{2 n-1} e_{n l}(x) \frac{\partial}{\partial x_{l}} .
$$

In terms of dilated coordinates $y$ in $W_{b}\left(x_{0}\right)$, we set $E_{k l}=e_{k l} \cdot D_{\varepsilon, x_{0}}^{-1}, R_{k}=r_{k} \cdot D_{\varepsilon, x_{0}}^{-1}$, $\Phi=\varphi \cdot D_{\varepsilon, x_{0}}^{-1}$ and $\Phi_{l}=\left(\partial \varphi / \partial x_{l}\right) \cdot D_{\varepsilon, x_{0}}^{-1}$.

By a direct calculation, one obtains that

$$
R_{k}=\frac{2 \varepsilon m \sum_{l=1}^{2 n-1} E_{v, l} \Phi^{2 m-1} \Phi_{l} y_{2 n}}{1+2 \varepsilon m \sum_{l=1}^{2 n-1} E_{n, l} \Phi^{2 n-1} \Phi_{l} y_{2 n}}, \quad 1 \leq k \leq n-1 .
$$

We set

$$
|f|_{k, W_{b}\left(x_{0}\right)}=\sup \left\{\left|D_{y}^{\alpha} f(y)\right| ; y \in W_{b}\left(x_{0}\right),|\alpha| \leq k\right\},
$$

and extend this norm to vector fields and 1-forms by using the coefficients of $\partial / \partial y_{j}$ or $d y_{j}$. By virtue of Proposition 3.2, it follows that for all $x \in W_{b}\left(x_{0}\right)$, we have

$$
\mu(x, \delta) \approx \tau\left(x_{0}, \delta\right) \lesssim \delta^{1 / m}=\varepsilon^{1 / m} \varphi\left(x_{0}\right)^{2} .
$$

Combining (4.10) with (4.11), we conclude that for each $s \geq 0$ there are $C_{s}$ independent of $x_{0}$ and $\delta$ such that

$$
\left|\delta^{-1} \mu(x, \delta) R_{k}\right|_{s, W_{b}\left(x_{0}\right)} \leq C_{s} b \varepsilon^{1 / m}, \quad 1 \leq k \leq n-1 .
$$


Therefore $L_{1}$ can be written, in $y$-coordinates, as

$$
\begin{aligned}
L_{1}= & \frac{\mu(x, \delta)}{\mu\left(x_{0}, \delta\right)} \sum_{l=1}^{2} E_{1, l} \frac{\partial}{\partial y_{l}}+\mu(x, \delta) \varepsilon^{-1 / 2} \varphi\left(x_{0}\right)^{-m} \sum_{l=3}^{2 n-2} E_{1, l} \frac{\partial}{\partial y_{l}} \\
& +\mu(x, \delta) \varepsilon^{-1} \varphi\left(x_{0}\right)^{-2 m} E_{1,2 n-1} \frac{\partial}{\partial y_{2 n-1}}+\mathcal{O}(\tilde{E}),
\end{aligned}
$$

where

$$
\begin{array}{ll}
\left|E_{1,1}-1\right|_{s, W_{b}\left(x_{0}\right)} \leq C \mu\left(x_{0}, \delta\right), & s \leq m, \\
\left|E_{1,2}+i\right|_{s, W_{b}\left(x_{0}\right)} \leq C \mu\left(x_{0}, \delta\right), & s \leq m,
\end{array}
$$

and $\tilde{E}$ satisfies, from the estimates in (4.12), that

$$
|\tilde{E}|_{s, W_{b}\left(x_{0}\right)} \leq C \varepsilon^{1 / m}, \quad s \leq m,
$$

for an independent constannt $C>0$. By virtue of the estimates in (3.31), we also have

(4.16) $\left|\mu(x, \delta) \varepsilon^{-1 / 2} \varphi\left(x_{0}\right)^{-m} E_{1, l}\right|_{k, W_{b}\left(x_{0}\right)} \leq C \tau\left(x_{0}, \delta\right)^{\gamma}, \quad k \leq m+1, \quad 3 \leq l \leq 2 n-2$.

Observe that the diameter in the $x$-coordinates of $W_{b}\left(x_{0}\right)$ is $\mathcal{O}\left(b \mu\left(x_{0}, \delta\right)\right) \ll \varphi\left(x_{0}\right)$ by (4.11). Hence it is clear that $\mu(x, \delta) \mu\left(x_{0}, \delta\right)^{-1}$ and $\Phi \varphi\left(x_{0}\right)^{-1}$ are very close to 1 (independent of $x_{0}$ and $\left.\delta\right)$ in $W_{b}\left(x_{0}\right)$ if $b$ is small. We also observe that $\mu(x, \delta)$ is defined independently of coordinate functions and that $\tau\left(x_{0}, \delta\right) \approx \tau(x, \delta) \approx \mu(x, \delta)$ if $x \in W_{b}\left(x_{0}\right)$. Therefore it follows that $\mu\left(x_{0}, \delta\right) \approx \mu\left(p_{0}, \delta\right)$ and $\varphi\left(x_{0}\right) \approx \varphi\left(p_{0}\right)$ if $W_{b}\left(x_{0}\right) \cap W_{b}\left(p_{0}\right) \neq 0$. These facts prove (ii).

Now set

$$
w_{k}=\frac{1}{2}\left(y_{2 k-1}-i y_{2 k}\right), \quad 1 \leq k \leq n-1, \quad \text { and } \quad w_{n}=\frac{1}{2}\left(y_{2 n}-i y_{2 n-1}\right),
$$

and define $D_{l}=\partial / \partial y_{l}, 1 \leq l \leq 2 n$, and $B_{m-1}(y)=b_{m-1} \circ D_{\varepsilon, x_{0}}^{-1}(y)$, where $b_{m-1}$ is the $(m-1)$-th order polynomial of $b(\zeta)$ in $\zeta_{1}$ and $\bar{\zeta}_{1}$ as in (3.6). We recall that the real part $e(\zeta)$ of the coefficient function of $\partial / \partial x_{2 n-1}$ in $L_{1}$ satisfies the estimates in (3.5). Hence, by combining (4.12)-(4.16), we obtain that

$$
\begin{aligned}
& L_{1}=\frac{\partial}{\partial w_{1}}+\mu\left(x_{0}, \delta\right) \delta^{-1} B_{m-1}(y) \frac{\partial}{\partial y_{2 n-1}}+\mathcal{O}(E), \\
& X=L_{1}+\bar{L}_{1}=\frac{\partial}{\partial y_{1}}+\mathcal{O}(E),
\end{aligned}
$$

where

$$
|E|_{s, W_{b}\left(x_{0}\right)} \leq C_{s} \varepsilon^{\gamma / m}, \quad\left|\mu\left(x_{0}, \delta\right) \delta^{-1} B_{m-1}\right|_{s, W_{b}\left(x_{0}\right)} \leq C_{s}
$$

for an independent constant $C>0$. Combining (4.17)-(4.19), we conclude that if $b \leq \sqrt{\sigma}$,

$$
\lim _{\sigma \rightarrow 0}\left|L_{1}-\left(\frac{\partial}{\partial w_{1}}+\mu\left(x_{0}, \delta\right) \delta^{-1} B_{m-1}(y) \frac{\partial}{\partial y_{2 n-1}}\right)\right|_{s, W_{b}\left(x_{0}\right)}=0, \quad s \leq m+1 .
$$

Setting $W\left(x_{0}\right)=W_{\sigma}\left(x_{0}\right)$ for sufficiently small $\sigma$, we obtain (i) and (iii). 
To prove (iv), we recall that $L_{n}=\partial / \partial y_{2 n}-i \partial / \partial y_{2 n-1}+y_{2 n} T$, where $T y_{2 n} \equiv 0$, and that $\mathcal{L}$ is integrable to infinite order along $M_{0}$. Hence

$$
c_{1 n}^{n}=\omega^{n}\left(\left[L_{1}, \bar{L}_{n}\right]\right)=\omega^{n}\left(\left[L_{1}+\bar{L}_{1}, \frac{\partial}{\partial y_{2 n}}-i \frac{\partial}{\partial y_{2 n-1}}\right]\right)+\mathcal{O}\left(y_{2 n}\right) .
$$

Combining (4.18)-(4.20), we conclude that

$$
\sup _{y \in W\left(x_{0}\right)}\left|c_{1 n}^{n}(y)\right| \leq C_{1}\left(\varepsilon^{\gamma / m}+\sigma^{2 m}\right),
$$

where $\gamma=(10 \times(m / 2) !)^{-1}$.

For the estimates of $c_{1 l}^{n}, l=2, \ldots, n-1$, we need the estimates in (3.26). Then it follows that $\left|c_{1 l}^{n}(y)\right| \leq C_{1} \varepsilon^{\gamma / m}$, along $M_{0}$. Since $L_{1}$ and $L_{l}$ are tangential vector fields, for $2 \leq l \leq n-1$, it follows that

$$
\left|c_{1 l}^{n}(y)\right| \leq C_{1}\left(\varepsilon^{\gamma / m}+\left|y_{2 n}\right|\right) \leq C_{1}\left(\varepsilon^{\gamma / m}+\sigma^{2 m}\right), \quad y \in W\left(x_{0}\right),
$$

Now we assume that $\varepsilon \leq \sigma^{2 m^{2} \cdot \gamma^{-1}}$. Then (4.2) follows from (4.21) and (4.22).

By Proposition 3.5, it follows that $\tau\left(x_{0}, \delta\right) \approx \mu(x, \delta)$ for $x \in W\left(x_{0}\right)$. Since $\left\{L_{1}, \ldots, L_{n}\right\}$ is orthonormal with respect to $\langle$,$\rangle , we conclude that (4.3) and (4.4) hold if \sigma$ is sufficiently small.

Using the special coordinates $y_{1}, \ldots, y_{2 n}$ and the special frames $L_{1}, \ldots, L_{n}$ defined in (4.9), we want to define $L^{2}$-operators with mixed boundary conditions. In the process of subelliptic estimates for $D_{2}$-operator, we will see that certain boundary integral terms on $M_{0}$ occur. To handle these boundary integral terms, we need the following lemma.

LEMmA 4.3. There are a frame $\left\{X_{1}, \ldots, X_{n}\right\}$ for $\mathcal{L}$ and its dual frame $\left\{\eta^{1}, \ldots, \eta^{n}\right\}$ so that if we set $c_{k n}^{n}=\eta^{n}\left(\left[X_{k}, \bar{X}_{n}\right]\right), 1 \leq k \leq n-1$, then

$$
\begin{aligned}
& c_{k n}^{n}=0 \text { on } W\left(x_{0}\right), \quad k=2, \ldots, n-1, \\
& \left|c_{1 n}^{n}\right|_{s, W\left(x_{0}\right) \leq C_{s} \sigma^{2 m} .}
\end{aligned}
$$

Proof. With the frames $L_{1}, \ldots, L_{n}$ and $\omega^{1}, \ldots, \omega^{n}$ defined on $W\left(x_{0}\right)$ in (4.9), we let $b_{k}(y), 2 \leq k \leq n-1$, be the smooth function satisfying

$$
\omega^{n}\left(\left[L_{k}, \bar{L}_{n}\right]\right)(y)+\sum_{l=2}^{n-1} \bar{b}_{l}(y) \omega^{n}\left(\left[L_{k}, \bar{L}_{l}\right]\right)(y) \equiv 0, \quad y \in W\left(x_{0}\right) .
$$

Since the Levi-form of $L_{2}, \ldots, L_{n-1}$ is always positive definite, (4.24) is solvable on $W\left(x_{0}\right)$. Set

$$
X_{k}=L_{k}, \quad k=1, \ldots, n-1, \quad \text { and } \quad X_{n}=L_{n}+\sum_{l=2}^{n-1} b_{l}(y) L_{l} .
$$

Then its dual frames $\eta^{k}, k=1, \ldots, n$, are given by

$$
\eta^{k}=\omega^{k}-b_{k}(y) \omega^{n}, \quad k=1, \ldots, n-1, \text { and } \eta^{n}=\omega^{n} .
$$


In terms of the new frames it follows from (4.24) that

$$
c_{k n}^{n}:=\eta^{n}\left(\left[X_{k}, \bar{X}_{n}\right]\right)=\omega^{n}\left(\left[L_{k}, \bar{L}_{n}\right]\right)+\sum_{l=2}^{n-1} \bar{b}_{l} \omega^{n}\left(\left[L_{k}, \bar{L}_{n}\right]\right) \equiv 0, \quad 2 \leq k \leq n-1,
$$

on $W\left(x_{0}\right)$ and

$$
c_{1 n}^{n}:=\omega^{n}\left(\left[X_{1}, \bar{X}_{n}\right]\right)=\omega^{n}\left(\left[L_{1}, \bar{L}_{n}\right]\right)+\sum_{l=2}^{n-1} \bar{b}_{l}(y) \omega^{n}\left(\left[L_{1}, \bar{L}_{l}\right]\right) .
$$

Therefore (4.23) follows from (4.25) and (4.2).

Recall that a deformation of $\mathcal{L}^{0}$ is a section $A$ of the bundle $\Gamma^{1}\left(S_{\varepsilon, \sigma}\right)$. In terms of the special frames in $W\left(x_{0}\right)$, we write $A=\sum_{j, l=1}^{n} A_{j l} \bar{\omega}^{l} \otimes L_{j}$, and then define

$$
\begin{aligned}
|A(y)|_{s} & =\sum_{|\alpha| \leq s} \sum_{j, l=1}^{n}\left|D_{y}^{\alpha} A_{j l}(y)\right|, \\
|A|_{s, W\left(x_{0}\right)} & =\sup \left\{|A(y)|_{s} ; y \in W\left(x_{0}\right)\right\} .
\end{aligned}
$$

We suppose that $A$ satisfies

$$
|A|_{m+2 n+3, W\left(x_{0}\right)} \leq \varepsilon_{0}
$$

for a sufficiently small $\varepsilon_{0}>0$.

We define $\mathcal{A}\left(S_{\varepsilon, \sigma}\right)$ to be the space of sections $A \in \Gamma^{0,1}\left(S_{\varepsilon, \sigma} ; 0\right)$ such that along $M_{0}$, $A(\bar{L})=0$ whenever $\bar{L} \in T^{0,1} \cap \boldsymbol{C} T M_{0}$. From now on, we assume that $A \in \mathcal{A}\left(S_{\varepsilon, \sigma}\right)$. Then we can define a deformation $\mathcal{L}^{A}$ of $\mathcal{L}^{0}$ by

$$
\overline{\mathcal{L}}^{A}=\left\{\bar{L}+A(\bar{L}) ; \bar{L} \in \overline{\mathcal{L}_{z}^{0}}, z \in S_{\varepsilon, \sigma}\right\} .
$$

In terms of the frames $X_{1}, \ldots, X_{n}$, and its dual frames $\eta^{1}, \ldots, \eta^{n}$ in $W\left(x_{0}\right)$ constructed in Lemma 4.3, we define

$$
X_{j}^{A}=X_{j}+\bar{A}\left(X_{j}\right), \quad j=1, \ldots, n
$$

and let $\eta_{A}^{j}$ be the dual frames. Set

$$
L_{j}^{A}=\sigma^{1 / 4}\left(X_{j}^{A}-\left(X_{j}^{A} r\right)\left(X_{n}^{A} r\right)^{-1} X_{n}^{A}\right), \quad 1 \leq j \leq n-1, \quad L_{n}^{A}=X_{n}^{A}
$$

and

$$
\omega_{A}^{j}=\sigma^{-1 / 4} \eta_{A}^{j}, \quad 1 \leq j \leq n-1, \quad \omega_{A}^{n}=\left(\eta_{A}^{n}+\sum_{j=1}^{n-1}\left(X_{j}^{A} r\right)\left(X_{n}^{A} r\right)^{-1} \eta_{A}^{j}\right)
$$

Obviously, the frames $\omega_{A}^{j}$, for $j=1, \ldots, n$, are dual to $L_{j}^{A}$, and $L_{j}^{A} r \equiv 0$ for $1 \leq j \leq n-1$. 
Note that we can write $L_{n}=\partial / \partial y_{2 n}-i \partial / \partial y_{2 n-1}+y_{2 n} T$, where $T y_{2 n} \equiv 0$. Assuming that $A$ satisfies (4.26) for sufficiently small $\varepsilon_{0} \leq \sigma^{2 m^{2} \cdot \gamma^{-1}}$, it follows from Lemma 4.3 that

$$
\begin{aligned}
& \sup _{y \in W\left(x_{0}\right)}\left|\omega_{A}^{n}\left(\left[L_{k}^{A}, \bar{L}_{n}^{A}\right]\right)(y)\right| \leq C \sigma^{1 / 4}|A|_{1}, \quad 2 \leq k \leq n-1, \\
& \sup _{y \in W\left(x_{0}\right)}\left|\omega_{A}^{n}\left(\left[L_{1}^{A}, \bar{L}_{n}^{A}\right]\right)(y)\right| \leq C \sigma^{2 m+1 / 4},
\end{aligned}
$$

where the constant $C>0$ is independent of $x_{0}, \sigma$ and $\varepsilon$.

In order to measure how $L_{j}^{A}, j=1,2, \ldots, n$, depend on $A$, we define

$$
P_{k}(y ; A)=\sum_{\substack{k_{1}, \ldots, k_{N} \\\left|k_{1}\right|+\cdots+\left|k_{N}\right| \leq k}} \prod_{\nu=1}^{N}|A(y)|_{k_{\nu}} .
$$

In the sequel, we assume that $A$ satisfies (4.26) for sufficiently small $\varepsilon_{0}$.

LEMMA 4.4. For $y \in W\left(x_{0}\right)$, the following pointwise estimates hold:

$$
\left|L_{k}^{A}-\sigma^{1 / 4} L_{k}\right|_{s} \leq C_{s} P_{s}(y ; A), \quad\left|\omega_{A}^{k}-\sigma^{-1 / 4} \omega^{k}\right|_{s} \leq C_{s} \sigma^{-1 / 4} P_{s}(y ; A)
$$

for $1 \leq k \leq n-1$ and

$$
\left|L_{n}^{A}-L_{n}\right|_{s} \leq C_{S} P_{S}(y ; A), \quad\left|\omega_{A}^{n}-\omega^{n}\right|_{s} \leq C_{S} P_{S}(y ; A) .
$$

Proof. From the expression of $L_{k}^{A}$ and $\omega_{A}^{k}$ in (4.27) and (4.28), the error terms are the finite product of derivatives as in (4.31) and (4.32).

For the subelliptic estimates on the non-euclidean balls $W\left(x_{0}\right)$, we still have to construct a family of plurisubharmonic functions with maximal Hessian in dilated coordinates $y$ defined in (4.7). By virtue of Theorem 3.6 there is a family of plurisubharmonic functions $\left\{\psi_{\delta}(x)\right\}_{\delta>0}$ defined on $\Omega_{\delta} \cap U=\left\{\left(x^{\prime}, t\right) ; t \leq \delta\right\} \cap U$. We may assume that there is an open set $\tilde{W}\left(x_{0}\right)=W_{C \sigma}\left(x_{0}\right)$, for some $C>1$, such that $W\left(x_{0}\right)=W_{\sigma}\left(x_{0}\right) \Subset \tilde{W}\left(x_{0}\right) \Subset P_{a \delta}\left(x_{0}\right)$, provided $\sigma$ is sufficiently small. We define

$$
S_{\rho}=\left\{y \in \Omega_{\rho} \cap U ;\left|y_{2 n}\right|<\rho\right\},
$$

and set $\delta_{0}=\delta\left(x_{0}\right)=\varepsilon \phi\left(x_{0}\right)^{2 m}$ and

$$
\mu_{1}(x)=\mu\left(x, \delta_{0}\right), \quad \mu_{k}(x)=\varepsilon^{1 / 2} \varphi(x)^{m}, 2 \leq k \leq n-1, \quad \mu_{n}(x)=\varepsilon \varphi(x)^{2 m} .
$$

For any $\rho>0$ we set

(4.34) $\quad \mu_{1}^{\rho}(x)=\mu\left(x, \rho \delta_{0}\right), \quad \mu_{k}^{\rho}(x)=\left(\rho \delta_{0}\right)^{1 / 2}, 2 \leq k \leq n-1, \quad \mu_{n}^{\rho}(x)=\rho \delta_{0}$.

THEOREM 4.5. For each small $\rho>0$, there exists a $C^{\infty}$ plurisubharmonic function $\lambda_{\rho}$ defined on $\tilde{W}\left(x_{0}\right) \ni W\left(x_{0}\right)$ satisfying the following:

(i) $\left|\lambda_{\rho}\right| \leq 1$ in $\tilde{W}\left(x_{0}\right)$.

(ii) For all $y \in S_{\rho} \cap \tilde{W}\left(x_{0}\right)$, and $L^{A}=\sum_{j=1}^{n} b_{j} L_{j}^{A}$, we have

$$
\partial \bar{\partial} \lambda_{\rho}(y)\left(L^{A}, \bar{L}^{A}\right) \approx \sum_{k=1}^{n}\left|b_{k}\right|^{2} \mu_{k}^{2}\left(\mu_{k}^{\rho}\right)^{-2} .
$$


(iii) $\left|L^{A} \lambda_{\rho}\right|^{2} \lesssim \partial \bar{\partial} \lambda_{\rho}(y)\left(L^{A}, \bar{L}^{A}\right)$.

(iv) $\left|D^{\alpha} \lambda_{\rho}\right| \leq C_{\alpha} \prod_{k=1}^{n} \mu_{k}^{\alpha_{i}}(x)\left(\mu_{k}^{\rho}\right)^{-\alpha_{i}}$, where $D^{\alpha}=\partial_{1}^{\beta_{1}} \bar{\partial}_{1}^{\gamma_{1}} \cdots \partial_{n}^{\beta_{n}} \bar{\partial}_{n}^{\gamma_{n}}, \alpha_{i}=\beta_{i}+\gamma_{i}$.

PROOF. Let $\left\{\psi_{\rho \delta_{0}}\right\}_{\rho \delta_{0}>0}$ be the family of plurisubharmonic functions constructed in Theorem 3.6. Set $\lambda_{\rho}(y)=\psi_{\rho \delta_{0}} \circ D_{x_{0}}^{-1}(y)$, where $D_{x_{0}}$ is the dilation function defined in (4.8). It is clear that $\lambda_{\rho}$ is plurisubharmonic and satisfies (i). Note that the orthonormal frame $\left\{L_{1}, \ldots, L_{n}\right\}$ defined in (4.9) can be written as $L_{j}=\mu_{j}(x)\left(L_{j}^{v}-r_{j} L_{n}^{v}\right), 1 \leq j \leq n-1$, and $L_{n}=\mu_{n}(x) L_{n}^{v}$.

If we let $L=\sum_{j=1}^{n} b_{j} L_{j}$, then it follows by functoriality that

$$
\begin{aligned}
\partial \bar{\partial} \lambda_{\rho}(y)(L, \bar{L})= & \partial \bar{\partial} \psi_{\rho \delta_{0}}(x)\left(d D_{x_{0}}^{-1} L, d D_{x_{0}}^{-1} \bar{L}\right) \\
= & \partial \bar{\partial} \psi_{\rho \delta_{0}}(x)\left(\sum_{j=1}^{n} b_{j} \mu_{j} L_{j}^{v}, \sum_{k=1}^{n} \bar{b}_{k} \mu_{k} \bar{L}_{k}^{v}\right) \\
& -2 \operatorname{Re}\left[\sum_{j=1}^{n-1} \sum_{k=1}^{n} r_{j} b_{j} \bar{b}_{k} \mu_{j} \mu_{k} \partial \bar{\partial} \psi_{\rho \delta_{0}}(x)\left(L_{n}^{v}, \bar{L}_{k}^{v}\right)\right] \\
& +\sum_{j, k=1}^{n-1} r_{j} \bar{r}_{k} b_{j} \bar{b}_{k} \mu_{j} \mu_{k} \partial \bar{\partial} \psi_{\rho \delta_{0}}(x)\left(L_{n}^{v}, \bar{L}_{n}^{v}\right) .
\end{aligned}
$$

From the expression of $r_{k}(x)$ in (4.10) and (4.12) it follows that

$$
\left|r_{k}(x)\right| \lesssim \varepsilon^{1 / m}\left(\rho \delta_{0}\right) \cdot \mu\left(x, \delta_{0}\right)^{-1} \text { for }|t| \leq \rho \delta_{0} .
$$

If we combine (4.36) with the properties (ii) and (iii) of Theorem 3.6, we conclude that

$$
\partial \bar{\partial} \lambda_{\rho}(y)(L, \bar{L}) \approx \sum_{k=1}^{n}\left|b_{k}\right|^{2} \mu_{k}^{2}\left(\mu_{k}^{\rho}\right)^{-2} .
$$

Note that the vector fields $L_{j}^{A}$, s and its dual frames $\omega_{j}^{A}$,s were written in terms of $L_{j}$ 's, and $\eta_{A}^{j}$ 's as in (4.27) and (4.28). By virtue of the expressions in (4.31) and (4.32) we can write

$$
\partial \bar{\partial} \lambda_{\rho}(y)\left(L^{A}, \bar{L}^{A}\right)=\partial \bar{\partial} \lambda_{\rho}(y)(L, \bar{L})+\mathcal{O}\left(A^{2} \rho^{-2}+A \rho^{-3 / 2}\right)|L|^{2} .
$$

Recall that $A=0$ along $M$ and satisfies (4.26). Hence $|A|_{0} \lesssim \varepsilon_{0} \rho^{2}$ provided $\left|y_{2 n}\right| \leq \rho$. Therefore it follows from (4.37) and (4.38) that

$$
\partial \bar{\partial} \lambda_{\rho}(y)\left(L^{A}, \bar{L}^{A}\right) \approx \sum_{k=1}^{n}\left|b_{k}\right|^{2} \mu_{k}(x)^{2}\left(\mu_{k}^{\rho}\right)^{-2} .
$$

This proves (ii). (iii) follows from the estimate

$$
\left|L^{A} \lambda_{\rho}\right|^{2} \lesssim \sum_{k=1}^{n}\left|b_{k}\right|^{2}\left|L_{k}^{A} \lambda_{\rho}\right|^{2} \lesssim \sum_{k=1}^{n}\left|b_{k}\right|^{2} \mu_{k}(x)^{2}\left(\mu_{k}^{\rho}\right)^{-2} \approx \partial \bar{\partial} \lambda_{\rho}(y)\left(L^{A}, \bar{L}^{A}\right) .
$$

(iv) follows from the property (iii) of Theorem 3.6. 
Next, we show that there exists a smooth Hermitian metric on $S_{\varepsilon, \sigma}$ such that for all $x_{0} \in$ $M$ the frames $L_{1}^{A}, \ldots, L_{n}^{A}$ given by (4.27) are orthonormal. For $L \in \mathcal{L}^{0}$ and $A \in \mathcal{A}\left(S_{\varepsilon, \sigma}\right)$ satisfying (4.26), define a bundle isomorphism $P_{A}: \mathcal{L}^{0} \rightarrow \mathcal{L}^{A}$ by $P_{A}(L)=L+A(L)$. Define a homomorphism $H_{A}: \mathcal{L}^{A} \rightarrow \mathcal{R}^{A}$, where $\mathcal{R}^{A}=\left\{L \in \mathcal{L}^{A} ; L r=0\right\}$, by

$$
H_{A}(L)=L-\frac{L r}{X_{n}^{A} r} X_{n}^{A}=L-\frac{L y_{2 n}}{L_{n}^{A} y_{2 n}} L_{n}^{A} .
$$

Then $H_{A} \circ P_{A}$ is an isomorphism of $\mathcal{R}$ onto $\mathcal{R}^{A}$. We define a metric $\langle,\rangle_{A}$ on $\mathcal{L}^{A}$ by

$$
\begin{aligned}
& \left\langle\left(H_{A} \circ P_{A}\right) \bar{L}_{1},\left(H_{A} \circ P_{A}\right) \bar{L}_{2}\right\rangle_{A}=\left\langle\bar{L}_{1}, \bar{L}_{2}\right\rangle, \quad \bar{L}_{1}, \bar{L}_{2} \in \mathcal{R}, \\
& \left\langle L_{n}^{A}, L_{n}^{A}\right\rangle_{A}=1, \\
& \left\langle\left(H_{A} \circ P_{A}\right) \bar{L}_{1}, L_{n}^{A}\right\rangle_{A}=0, \quad \bar{L}_{1} \in \mathcal{R} .
\end{aligned}
$$

Note that $L_{n}^{A}$ is actually globally defined, so that the above conditions determine a metric on $\mathcal{L}^{A}$. Since $L_{j}, j=1,2, \ldots, n-1$, defined in (4.9), are an orthonormal basis of $\mathcal{L}$, it follows that $L_{j}^{A}=\left(H_{A} \circ P_{A}\right) L_{j}, j=1,2, \ldots, n-1$, are an orthonormal basis of $\mathcal{L}^{A}$ with respect to $\langle,\rangle_{A}$.

Let $d V$ denote the volume form associated with the Riemannian metric $\langle$,$\rangle . In the$ coordinates $\left(y_{1}, \ldots, y_{2 n}\right)$ in $W\left(x_{0}\right)$ we can write $d V=V(y) d y$, where $d y=d y_{1} \cdots d y_{2 n}$, and where $V$ satisfies

$$
|V|_{s, W\left(x_{0}\right)} \leq C_{s} \quad \text { and } \quad \inf _{y \in W\left(x_{0}\right)} V(y)>c>0,
$$

where $c$ is independent of $\sigma, \varepsilon$ and $x_{0}$. We will define the inner product for two functions $g$, $h \in C^{\infty}\left(S_{\varepsilon, \sigma}\right)$ by

$$
(g, h)=\int g \bar{h} d V .
$$

Let $\Lambda^{0, q}\left(S_{\varepsilon, \sigma} ; A\right)$ denote the space of $(0, q)$-forms with respect to $\mathcal{L}^{A}$ on $S_{\varepsilon, \sigma}$, and set

$$
\Gamma^{0, q}\left(S_{\varepsilon, \sigma} ; A\right)=\Lambda^{0, q}\left(S_{\varepsilon, \sigma} ; A\right) \otimes \mathcal{L}^{A} .
$$

Now let us define, for a given structure $\mathcal{L}^{A}$, where $A$ satisfying (4.26) for small $\varepsilon_{0}$, the $L^{2}$ operators corresponding to $D_{q}: \Gamma^{0, q} \rightarrow \Gamma^{0, q+1}$ and its adjoint. We define $\mathcal{E}_{c}^{0, q}\left(S_{\varepsilon, \sigma} ; A\right)$ to be the set of smooth sections $U$ of $\Gamma^{0, q}\left(S_{\varepsilon, \sigma} ; A\right)$ such that support of $U$ is a compact subset of $S_{\varepsilon, \sigma}$. Let $\mathcal{E}_{0}^{0, q}\left(S_{\varepsilon, \sigma} ; A\right)$ denote the set of sections of $\mathcal{E}_{c}^{0, q}\left(S_{\varepsilon, \sigma} ; A\right)$ with compact support in the interior of $S_{\varepsilon, \sigma}$. Suppose that $U=\sum_{l=1}^{n} \sum_{|J|=q} U_{l}^{J} \bar{\omega}_{A}^{J} \cdot L_{l}^{A}$ is an element of $\Gamma^{0, q}\left(S_{\varepsilon, \sigma} ; A\right)$ with compact support in $W\left(x_{0}\right)$. We define

$$
\|U\|^{2}=\int_{S_{\varepsilon, \sigma}} \sum_{l=1}^{n} \sum_{|J|=q}\left|U_{l}^{J}\right|^{2} d V,
$$

where $d V$ is the volume form given by the metric of $\mathcal{L}^{0}$. Since $\left\{L_{1}^{A}, \ldots, L_{n}^{A}\right\}$ is an orthonormal frame, the quantity in (4.39) is independent of the frame neighborhood $W\left(x_{0}\right)$. Thus, by using a partition of unity, it follows that the norm in (4.39) extends to all of $\Gamma^{0, q}\left(S_{\varepsilon, \sigma} ; A\right)$. Let $L_{q}^{2}\left(S_{\varepsilon, \sigma}, T_{A}^{1,0}\right)$ denote the set of sections of $\Gamma^{0, q}\left(S_{\varepsilon, \sigma} ; A\right)$ such that (4.39) is finite. 
Define $\mathcal{B}_{-}^{q}\left(S_{\varepsilon, \sigma} ; A\right)$ to be the set of forms in $\mathcal{E}_{c}^{0, q}\left(S_{\varepsilon, \sigma} ; A\right)$ such that $U_{l}^{J}$ vanishes on $M_{0}$ whenever $n \notin J$. (This is also independent of the frame neighborhood $W\left(x_{0}\right)$.) Similarly, define $\mathcal{B}_{+}^{q}\left(S_{\varepsilon, \sigma} ; A\right)$ to be the set of forms in $\mathcal{E}_{c}^{0, q}\left(S_{\varepsilon, \sigma} ; A\right)$ such that $U_{l}^{J}$ vanishes on $M_{\sigma}$ whenever $n \in J$. We now define the formal adjoint $D_{q}^{\prime}$ of $D_{q}$ on $\mathcal{E}_{c}^{0, q}\left(S_{\varepsilon, \sigma} ; A\right)$ by $D_{q}^{\prime} U=$ $G \in \mathcal{E}_{c}^{0, q-1}\left(S_{\varepsilon, \sigma} ; A\right)$ if for all $V \in \mathcal{E}_{0}^{0, q-1}\left(S_{\varepsilon, \sigma} ; A\right)$,

$$
\left(U, D_{q} V\right)=(G, V) \text {, }
$$

where $($,$) corresponds to the norm in (4.39). By combining (2.3) with (2.6) together with$ integration by parts, it follows that if $U=\sum_{v} U_{v} L_{v}^{A} \in \Gamma^{0, k}\left(S_{\varepsilon} ; A\right)$ is supported in $W\left(x_{0}\right)$, then

$$
\left.D_{k}^{\prime} U=\sum_{\nu}\left(\bar{\partial}^{*} U_{\nu}-\sum_{\mu} \sum_{j} \partial \overline{\omega_{A}^{\mu}}\left(L_{j}^{A}, \bar{L}_{v}^{A}\right)\left(\bar{L}_{j}^{A}\right\rfloor U_{\mu}\right)\right) L_{v}^{A}
$$

where

$$
\begin{aligned}
\bar{\partial}^{*} U_{v}= & -\sum_{|J|=k-1} \sum_{j=1}^{n}\left(L_{j}^{A} U_{v}^{j J}+e_{j} U_{v}^{j J}\right) \bar{\omega}_{A}^{J} \\
& -\sum_{|K|=k-2} \sum_{l=1}^{n} \sum_{i<j} \omega_{A}^{l}\left(\left[L_{i}^{A}, L_{j}^{A}\right]\right) U_{v}^{i j K} \bar{\omega}_{A}^{l K} .
\end{aligned}
$$

We now extend the definition of the operator $D_{q}$ and $D_{q}^{\prime}$ to the $L^{2}$-spaces. Define $L_{k}^{2}\left(S_{\varepsilon, \sigma} ; A\right)$ to be the set of all sections $U$ of $\Gamma^{0, k}\left(S_{\varepsilon, \sigma} ; A\right)$ for which $\|U\|^{2}<\infty$. We define an operator

$$
T: L_{q-1}^{2}\left(S_{\varepsilon, \sigma} ; T_{A}^{1,0}\right) \rightarrow L_{q}^{2}\left(S_{\varepsilon, \sigma} ; T_{A}^{1,0}\right)
$$

by the condition that $U \in \operatorname{Dom}(T)$ and $T U=F \in L_{q}^{2}\left(S_{\varepsilon, \sigma} ; T_{A}^{1,0}\right)$ if for all $V \in \mathcal{B}_{-}^{q}\left(S_{\varepsilon, \sigma} ; A\right)$, we have

$$
\left(U, D_{q}^{\prime} V\right)=(F, V) .
$$

Similarly, we can define $S: L_{q}^{2}\left(S_{\varepsilon, \sigma} ; T_{A}^{1,0}\right) \rightarrow L_{q+1}^{2}\left(S_{\varepsilon, \sigma} ; T_{A}^{1,0}\right)$. Note that these definitions imply that if $U \in \operatorname{Dom}(T)($ or $\operatorname{Dom}(S))$, then $T U=D_{q} U$ (or $S U=D_{q+1} U$ ) as in the sense of distribution theory. Let $T^{*}: L_{q}^{2}\left(S_{\varepsilon, \sigma} ; T_{A}^{1,0}\right) \rightarrow L_{q-1}^{2}\left(S_{\varepsilon, \sigma} ; T_{A}^{1,0}\right)$ and $S^{*}: L_{q+1}^{2}\left(S_{\varepsilon, \sigma} ; T_{A}^{1,0}\right) \rightarrow L_{q}^{2}\left(S_{\varepsilon, \sigma} ; T_{A}^{1,0}\right)$ be the Hilbert space adjoints of $T$ and $S$, respectively. It follows that if $U \in \operatorname{Dom}\left(T^{*}\right)$ and $V \in \operatorname{Dom}\left(S^{*}\right)$, then

$$
T^{*} U=D_{q}^{\prime}(U) \text { and } S^{*} V=D_{q+1}^{\prime}(V),
$$

as in the sense of distributions. Therefore it follows that

$$
\begin{aligned}
& \mathcal{E}_{c}^{0, q-1}\left(S_{\varepsilon, \sigma} ; A\right) \cap \operatorname{Dom}(T)=\mathcal{B}_{+}^{q-1}\left(S_{\varepsilon, \sigma} ; A\right), \\
& \mathcal{E}_{c}^{0, q}\left(S_{\varepsilon, \sigma} ; A\right) \cap \operatorname{Dom}\left(T^{*}\right)=\mathcal{B}_{-}^{q}\left(S_{\varepsilon, \sigma} ; A\right) .
\end{aligned}
$$

Similar relations hold for $S$. Set

$$
\mathcal{B}^{q}\left(S_{\varepsilon, \sigma} ; A\right)=\mathcal{B}_{+}^{q}\left(S_{\varepsilon, \sigma} ; A\right) \cap \mathcal{B}_{-}^{q}\left(S_{\varepsilon, \sigma} ; A\right) .
$$


Then we can approximate $U \in \operatorname{Dom}(S) \cap \operatorname{Dom}\left(T^{*}\right)$ by $U_{\mu} \in \mathcal{B}^{q}\left(S_{\varepsilon, \sigma} ; A\right)$ in the graph norm of $S$ and $T^{*}$ [5, Lemma 6.4].

Lemma 4.6. Let $U \in \operatorname{Dom}(S) \cap \operatorname{Dom}\left(T^{*}\right)$. Then there exists $U_{\mu} \in \mathcal{B}^{q}\left(S_{\varepsilon, \sigma} ; A\right)$ such that

$$
\lim _{\mu \rightarrow \infty}\left(\left\|U_{\mu}-U\right\|+\left\|S U_{\mu}-S U\right\|+\left\|T^{*} U_{\mu}-T^{*} U\right\|\right)=0 .
$$

Finally, suppose that we have proved the estimate

$$
\|U\|^{2} \leq C\left(\left\|T^{*} U\right\|^{2}+\|S U\|^{2}\right)
$$

for all $U \in \mathcal{B}^{q}\left(S_{\varepsilon, \sigma} ; A\right)$. Then Lemma 4.6 shows that (4.43) holds for all $U \in \operatorname{Dom} T^{*} \cap$ Dom $S$. Then from the usual $\bar{\partial}$-Neumann theory it follows that for all $G \in L_{q}^{2}\left(S_{\varepsilon, \sigma} ; T_{A}^{1,0}\right)$, there exists an element $N G \in \operatorname{Dom}\left(T^{*}\right) \cap \operatorname{Dom}(S)$ such that

$$
\|N G\| \leq C^{2}\|G\|,
$$

and

$$
(G, V)=\left(T^{*}(N G), T^{*} V\right)+(S N G, S V), \quad V \in \operatorname{Dom}\left(T^{*}\right) \cap \operatorname{Dom}(S) .
$$

We will call $N$ the Neumann operator associated with $D_{q}$.

5. The subelliptic estimate for $D_{q}$. In this section we prove a subelliptic estimate for the $D_{q}$-Neumann problem with almost complex structure $\mathcal{L}^{A}$. We set $q=2$ in this section.

We first define tangential norms that will be used in the estimates. For any $s \in \boldsymbol{R}$, set

$$
\|\left.|f|\right|_{s} ^{2}=\int_{0}^{\sigma^{2 m}} \int_{\boldsymbol{R}^{2 n-1}}\left|\hat{f}\left(\xi, y_{2 n}\right)\right|^{2}\left(1+|\xi|^{2}\right)^{s} d \xi d y_{2 n},
$$

where $\hat{f}\left(\xi, y_{2 n}\right)=\int_{\boldsymbol{R}^{2 n-1}} e^{-i y^{\prime} \cdot \xi} f\left(y^{\prime}, y_{2 n}\right) d y^{\prime}$. For any integer $k \geq 0$ and any $s \in \boldsymbol{R}$, set

$$
\|f\|_{s, k}^{2}=\left.\sum_{j=0}^{k}\left\|\frac{\partial^{j} f}{\partial y_{2 n}^{j}}\right\|\right|_{s-j} ^{2} .
$$

Then, for any integer $m \geq 0$ and $f \in C^{\infty}\left(W\left(x^{\prime}\right)\right)$, set

$$
\|f\|_{m}^{2}=\sum_{|\alpha| \leq m}\left\|D_{y}^{\alpha} f\right\|^{2}
$$

By using the coefficients of $U$, we can easily define all of the above norms for any section $U$ of $\Gamma^{0, q}$. We recall that $\mathcal{A}\left(S_{\varepsilon, \sigma}\right)$ is the space of sections $A \in \Gamma^{0,1}\left(S_{\varepsilon, \sigma} ; 0\right)$ such that along $M_{0}, A(\bar{L})=0$ whenever $\bar{L} \in T^{0,1} \cap C T M_{0}$. We let $C>1$ and $0<c \leq 1$ be independent constants which may vary in various estimations. Then the goal of this section is to prove the following subelliptic estimate:

Theorem 5.1. Suppose $T(\bar{M})=m<\infty$ and that $A$ is a section of $\mathcal{A}\left(S_{\varepsilon, \sigma}\right)$. Then there exist small positive constants $\sigma_{1}$ and $\varepsilon_{1}$ so that if $\varepsilon<\varepsilon_{1}, \sigma<\sigma_{1}$, and $|A|_{m+2 n+3, W\left(x_{0}\right)} \leq$ $\varepsilon$, then the $D_{q}$-Neumann problem on $S_{\varepsilon, \sigma}$ with coefficient $\sigma$ for the almost complex structure 
$\mathcal{L}^{A}$ satisfies the following estimate for all forms $U \in \mathcal{B}^{q}\left(S_{\varepsilon, \sigma} ; A\right)$ that are compactly supported in $W\left(x_{0}\right)$ :

$$
\sigma^{-3}\|U\|^{2}+L^{A}(U)+\sigma^{1 / 2}\|U\|_{1 / m, 1}^{2} \leq C\left(\|S U\|^{2}+\left\|T^{*} U\right\|^{2}\right),
$$

where $L^{A}(U)$ is defined by

$$
\begin{aligned}
L^{A}(U)= & \sum_{k=1}^{n-1} \sum_{l=1}^{n} \sum_{|J|=q}\left\|\bar{L}_{k}^{A} U_{l}^{J}\right\|^{2}+\sum_{k=1}^{n-1} \sum_{l=1}^{n} \sum_{\substack{|J|=q \\
n \notin J}}\left\|L_{k}^{A} U_{l}^{J}\right\|^{2} \\
& +\sum_{l=1}^{n} \sum_{\substack{|J|=q \\
n \in J}}\left\|L_{n}^{A} U_{l}^{J}\right\|^{2}+\sum_{l=1}^{n} \sum_{\substack{|J|=q \\
n \notin J}} \sqrt{\| L_{n}^{A}} U_{l}^{J} \|^{2} .
\end{aligned}
$$

We first state some necessary lemmas for the proof of Theorem 5.1 [5, Lemma 7.5].

LEMmA 5.2. Let $X_{j}=\sum_{k} a_{j k} \partial / \partial x_{k}, j=1, \ldots, l$, be smooth compactly supported vector fields in $\boldsymbol{R}^{d}$, and suppose that there exist a set $K \Subset \boldsymbol{R}^{d}$ and a constant $c>0$ such that for all $x \in K$,

$$
c<\inf \left\{\sum_{j}\left|\eta\left(X_{j}\right)\right|+\sum_{i<j}\left|\eta\left(\left[X_{i}, X_{j}\right]\right)\right| ; \eta \in T_{x}^{*},|\eta|=1\right\} .
$$

Then there exists a constant $C$ independent of $X_{1}, \ldots, X_{l}$ such that for all $u \in C_{0}^{\infty}\left(\boldsymbol{R}^{d}\right)$ with $\operatorname{supp} u \subset K$ and any integer $s>(d+5) / 2$,

$$
c\|u\|_{1 / 2}^{2} \leq C\left[\sum_{j=1}^{l}\left\|X_{j} u\right\|^{2}+\|u\|^{2} \sum_{j, k}\left\|a_{j, k}\right\|_{s}^{2}\right] .
$$

If $n \geq 3$, then the (n-2) positive eigenvalue condition on the Levi-form of $M$ guarantees the existence of at least one positive eigenvalue. Set $X_{2 k-1}=\operatorname{Re} L_{k}^{A}$ and $X_{2 k}=\operatorname{Im} L_{k}^{A}$ for $1 \leq k \leq n-1$. Then Lemma 5.2 and the expression of $L_{k}^{A}$ in (4.27) show that

Lemma 5.3. Assume that $n \geq 3$. Then for all $f \in C_{0}^{\infty}\left(W\left(x_{0}\right)\right)$,

$$
\sigma^{1 / 2}\||f|\|_{1 / 2}^{2} \leq C \sum_{k=1}^{n-1}\left(\left\|L_{k}^{A} f\right\|^{2}+\left\|\overline{L_{k}^{A}} f\right\|^{2}\right)+C\|f\|^{2} .
$$

For convenience, in what that follows we omit the notation $A$ from the frames $L_{1}^{A}, \ldots$, $L_{n}^{A}$, and $\omega_{A}^{1}, \ldots, \omega_{A}^{n}$, and $L^{A}$. Note that in $W\left(x_{0}\right)$, we have technically chosen coordinates in such a way that $y_{2 n}=0$ and $y_{2 n}=\sigma^{2 m}$ coincide with $r=0$ and $r=\varepsilon \sigma^{2 m}$, respectively, on the boundaries of $S_{\varepsilon, \sigma}$. Then the following lemma can be proved by modifying the proof of Lemma 7.7 in [5].

Lemma 5.4. Suppose that $f \in C_{0}^{\infty}\left(W\left(x_{0}\right)\right)$ and $f$ vanishes either on $M_{0}$ or on $M_{\sigma}$. If $\sigma$ is sufficiently small, say $\sigma<\sigma_{1}$, then there exists a constant $C$ independent of $\varepsilon, \sigma$, and 
$x_{0}$ such that

$$
\sigma^{-4}\|f\|^{2} \leq C\left(\|f\|_{1 / m}^{2}+\left\|\tilde{L}_{n} f\right\|^{2}\right)
$$

where $\tilde{L}_{n}=L_{n}$ or $\bar{L}_{n}$.

To handle the commutator terms, we need the following lemma.

Lemma 5.5. Assume that $n \geq 3$. Let $U \in \mathcal{B}_{q}\left(S_{\varepsilon, \sigma} ; A\right)$ be compactly supported in $W\left(x_{0}\right)$ and assume that $|K|=q-1$ with $n \notin K$ and that $1 \leq k \leq n-1$. Set $c_{k n}^{n}=$ $w^{n}\left(\left[L_{k}, \bar{L}_{n}\right]\right)$ and $d_{n k}^{n}=\bar{\omega}^{n}\left(\left[L_{n}, \bar{L}_{k}\right]\right)$. Then

$$
\begin{aligned}
& \left|\left(c_{k n}^{n} L_{n} U_{l}^{k K}, U_{l}^{n K}\right)\right| \leq C\left(\sigma L^{A}(U)+\sigma^{-1}\|U\|^{2}\right), \\
& \left|\left(d_{k n}^{n} \bar{L}_{n} U_{l}^{n K}, U_{l}^{k K}\right)\right| \leq C\left(\sigma L^{A}(U)+\sigma^{-1}\|U\|^{2}\right) .
\end{aligned}
$$

Proof. Note that $U_{l}^{k K}=0$ on $M_{0}$ and $U_{l}^{n K}=0$ on $M_{\sigma}$. From (4.29) and (4.30), it follows that

$$
\left|c_{k n}^{n}\right|_{0},\left|d_{n k}^{n}\right|_{0} \leq C \sigma^{2 m}, \quad 1 \leq k \leq n-1 .
$$

Let $\chi$ be a $C^{\infty}$ function defined on $\tilde{W}\left(x_{0}\right) \ni W\left(x_{0}\right)$ such that $0 \leq \chi \leq 1, \chi=0$ near $M_{0}$, $\chi=1$ near $M_{\sigma}$ and satisfies

$$
|\chi|_{s, \tilde{W}\left(x_{0}\right)} \leq C \sigma^{-2 m s}, \quad s=1,2, \ldots .
$$

Let us write

$$
\left(c_{k n}^{n} L_{n} U_{l}^{k K}, U_{l}^{n K}\right)=\left(c_{k n}^{n} \chi L_{n} U_{l}^{k K}, U_{l}^{n K}\right)+\left(c_{k n}^{n}(1-\chi) L_{n} U_{l}^{k K}, U_{l}^{n K}\right) .
$$

By integration by parts we get, from the estimates in (5.8), that

$$
\begin{aligned}
\left(c_{k n}^{n} \chi L_{n} U_{l}^{k K}, U_{l}^{n K}\right) & =-\left(U_{l}^{k K}, c_{k n}^{n} \chi \bar{L}_{n} U_{l}^{n K}\right)+\mathcal{O}\left(\|U\|^{2}\right) \\
& \lesssim \sigma \cdot \sigma^{4 m}\left\|\chi \bar{L}_{n} U_{l}^{n K}\right\|^{2}+\sigma^{-1}\|U\|^{2} .
\end{aligned}
$$

Using the fact that $\chi=0$ on $M_{0}, U_{l}^{n K}=0$ on $M_{\sigma}$, and (5.9), we can perform integration by parts for the function $\left\|\chi \bar{L}_{n} U_{l}^{n K}\right\|^{2}$ in a standard way. Then we get

$$
\left\|\chi \bar{L}_{n} U_{l}^{n K}\right\|^{2}=\left\|\chi L_{n} U_{l}^{n K}\right\|^{2}+\sigma^{-4 m} \mathcal{O}\left(\left\|U_{l}^{n K}\right\|^{2}+L(U)\right) .
$$

Combining (5.10) and (5.11), we get

$$
\left|\left(c_{k n}^{n} \chi L_{n} U_{l}^{k K}, U_{l}^{n K}\right)\right| \lesssim \sigma L^{A}(U)+\sigma^{-1}\|U\|^{2} .
$$

Similarly, we can estimate $\left(c_{k n}^{n}(1-\chi) L_{n} U_{l}^{k K}, U_{l}^{n K}\right)$. This proves (5.6). The proof of (5.7) is similar.

For each small $\rho>0$, we set

$$
S_{\rho}=\left\{\left(y^{\prime}, y_{n}\right) ;\left|y_{2 n}\right|<\rho\right\} \cap \tilde{W}\left(x_{0}\right),
$$

and let $\lambda_{\rho}=\psi_{\rho \delta_{o}} \circ D_{x_{0}}^{-1}(y)$ be the plurisubharmonic weight functions constructed in Theorem 4.5 , where $\delta_{0}=\varepsilon \phi\left(x_{0}\right)^{2 m}$. 
LEMMA 5.6. For each $k, 1 \leq k \leq n-1$, set $d_{1 k}^{n}=\bar{\omega}^{n}\left(\left[L_{1}, \bar{L}_{k}\right]\right)$ and let $x=D_{x_{0}}^{-1}(y)$. Then on $S_{\rho}$ we have

$$
\begin{aligned}
& \left|d_{11}^{n}\left(\bar{L}_{n} \lambda_{\rho}\right)(y)\right| \lesssim \sigma^{1 / 2} \tau\left(x, \delta_{0}\right)^{2} \tau\left(x, \rho \delta_{0}\right)^{-2}, \\
& \left|d_{1 k}^{n}\left(\bar{L}_{n} \lambda_{\rho}\right)(y)\right| \lesssim \sigma^{1 / 2} \rho^{-1 / 2} \tau\left(x, \delta_{0}\right) \tau\left(x, \rho \delta_{0}\right)^{-1+\gamma}, \quad 2 \leq k \leq n-1 .
\end{aligned}
$$

Proof. Note that $d_{1 k}^{n}=\bar{\omega}_{A}^{n}\left(\left[L_{1}^{A}, \bar{L}_{k}^{A}\right]\right)$, where $\omega_{A}^{n}$ and $L_{k}^{A}$ 's are defined in (4.27) and (4.28). Therefore it follows that

$$
d_{1 k}^{n}(y)=\sigma^{1 / 2} \omega^{n}\left(\left[L_{1}, \bar{L}_{k}\right]\right)(y)+\mathcal{O}\left(|A|_{1}\right) .
$$

Now assume that $y=\left(y^{\prime}, y_{2 n}\right) \in S_{\rho}$. Then $x=\left(x^{\prime}, t\right)=D_{x_{0}}^{-1}(y)$ satisfies

$$
|t| \leq \rho \varepsilon \phi\left(x_{0}\right)^{2 m}=\rho \delta_{0} .
$$

Let $\mu_{k}(x)$ be defined as in (4.33). Then, by functoriality, it follows that

$$
\begin{aligned}
\omega^{n} & \left(\left[L_{1}, \bar{L}_{k}\right]\right)(y)\left(\bar{L}_{n} \lambda_{\rho}\right)(y) \\
& =\mu_{n}(x)^{-1} \omega^{n}\left(\left[\mu_{1}(x) H\left(L_{1}^{v}\right), \mu_{k}(x) \overline{H\left(L_{k}^{v}\right)}\right]\right)(x) \mu_{n}(x) \bar{L}_{n}^{v} \psi_{\rho \delta_{0}}(x) \\
& =\mu_{1}(x) \mu_{k}(x) \omega^{n}\left(\left[L_{1}^{v}, \bar{L}_{k}^{v}\right]\right)(x) \bar{L}_{n}^{v} \psi_{\rho \delta_{0}}(x)+\mathcal{O}\left(x_{2 n} \bar{L}_{n}^{v} \psi_{\rho \delta_{0}}(x)\right),
\end{aligned}
$$

where we have used the notation in (4.9) and the expression of $r_{k}$ in (4.10).

Note that $\tau\left(x, \delta_{0}\right) \approx \mu\left(x, \delta_{0}\right) \approx \mu\left(x, \varepsilon \phi(x)^{2 m}\right)$, and $\mu_{k}(x) \approx \delta_{0}^{1 / 2}$ on $\tilde{W}\left(x_{0}\right)$. Since $x \in$ $S\left(\rho \delta_{0}\right)$ (in $x$-coordinates), it follows from Proposition 3.8 and the property (iii) of Theorem 3.6 that (set $\delta=\rho \delta_{0}$ there)

$$
\begin{aligned}
\left|\mu_{1}(x)^{2} \omega^{n}\left(\left[L_{1}^{v}, \bar{L}_{1}^{v}\right]\right)(x) \bar{L}_{n}^{v} \psi_{\rho \delta_{0}}(x)\right| & \lesssim \tau\left(x, \delta_{0}\right)^{2}\left(\rho \delta_{0}\right) \tau\left(x, \rho \delta_{0}\right)^{-2}\left(\rho \delta_{0}\right)^{-1} \\
& =\tau\left(x, \delta_{0}\right)^{2} \tau\left(x, \rho \delta_{0}\right)^{-2}
\end{aligned}
$$

and for $2 \leq k \leq n-1$, we have

$$
\begin{aligned}
& \left|\mu_{1}(x) \mu_{k}(x) \omega^{n}\left(\left[L_{1}^{\nu}, \bar{L}_{k}^{v}\right]\right)(x) L_{n}^{\nu} \psi_{\rho \delta_{0}}(x)\right| \\
& \quad \lesssim \tau\left(x, \delta_{0}\right) \delta_{0}^{1 / 2} \cdot\left(\rho \delta_{0}\right)^{1 / 2} \cdot \tau\left(x, \rho \delta_{0}\right)^{-1+\gamma}\left(\rho \delta_{0}\right)^{-1} \\
& \quad \lesssim \rho^{-1 / 2} \tau\left(x, \delta_{0}\right) \tau\left(x, \rho \delta_{0}\right)^{-1+\gamma} .
\end{aligned}
$$

Assuming that $A$ satisfies (4.26), it follows that $|A|_{1} \leq \varepsilon_{0} \rho^{2}$ if $y \in S_{\rho}$. Also, it follows from the property (iii) of Theorem 3.6 that

$$
\left|x_{2 n} \bar{L}_{n}^{\nu} \psi_{\rho \delta_{0}}(x)\right| \lesssim 1, \quad x \in S\left(\rho \delta_{0}\right)
$$

If we combine (5.14)-(5.17), then (5.12) and (5.13) follow.

We now want to prove Theorem 5.1. Assume $U=\sum_{l=1}^{n} \sum_{|J|=q} U_{l}^{J} \bar{\omega}^{J} \cdot L_{l} \in \mathcal{B}^{q}\left(S_{\varepsilon, \sigma} ; A\right)$ with supp $U \subset W\left(x_{0}\right)$. Then from (4.40) and (4.41) it follows that

$$
T^{*} U=D_{q}^{\prime} U=B U+C|U|
$$


where

$$
B U=-\sum_{l=1}^{n} \sum_{|K|=q-1} \sum_{j=1}^{n}\left(L_{j} U_{l}^{j K}\right) \bar{\omega}^{K} \cdot L_{l} .
$$

Also, (2.6) shows that

$$
S U=D_{q+1} U=A U+C|U|
$$

where

$$
A U=\sum_{l=1}^{n} \sum_{|J|=q} \sum_{j=1}^{n}\left(\bar{L}_{j} U_{l}^{J}\right) \bar{\omega}^{j J} \cdot L_{l}
$$

Combining (5.18)-(5.20), we see that

$$
\|A U\|^{2}+\|B U\|^{2} \leq 2\|S U\|^{2}+2\left\|T^{*} U\right\|^{2}+C\|U\|^{2} .
$$

Let us write $U=U^{\prime}+U^{\prime \prime}$, where

$$
U^{\prime}=\sum_{l=1}^{n} \sum_{\substack{|J|=q \\ n \in J}} U_{l}^{J} \bar{\omega}^{J} \cdot L_{l}, \quad U^{\prime \prime}=\sum_{l=1}^{n} \sum_{\substack{|J|=q \\ n \notin J}} U_{l}^{J} \bar{\omega}^{J} \cdot L_{l},
$$

and set

$$
\begin{aligned}
& L\left(U^{\prime}\right)=\sum_{l=1}^{n} \sum_{k=1}^{n-1} \sum_{\substack{|J|=q \\
n \in J}}\left\|\bar{L}_{k} U_{l}^{J}\right\|^{2}+\sum_{l=1}^{n} \sum_{\substack{|J|=q \\
n \in J}}\left\|L_{n} U_{l}^{J}\right\|^{2}, \\
& L\left(U^{\prime \prime}\right)=\sum_{l=1}^{n} \sum_{\substack{k=1 \\
k-1}}^{n}\left(\left\|\bar{L}_{k} U_{l}^{J}\right\|^{2}+\left\|L_{k} U_{l}^{J}\right\|^{2}\right)+\sum_{\substack{\mid=q \\
n \notin J}}^{n} \sum_{\substack{|J|=q \\
n \notin J}}\left\|\bar{L}_{n} U_{l}^{J}\right\|^{2} .
\end{aligned}
$$

Then we can write

$$
\|A U\|^{2}+\|B U\|^{2}=\left\|A U^{\prime \prime}\right\|^{2}+\left\|B U^{\prime \prime}\right\|^{2}+\left\|A U^{\prime}\right\|^{2}+\left\|B U^{\prime}\right\|^{2}+E\left(U^{\prime}, U^{\prime \prime}\right),
$$

where $E\left(U^{\prime}, U^{\prime \prime}\right)$ denotes the (sum of) inner products $\left(A U^{\prime}, A U^{\prime \prime}\right)$ and $\left(B U^{\prime}, B U^{\prime \prime}\right)$.

Note that the Levi-form of $M_{\sigma}$ has at least $(n-2)$-positive eigenvalues and $U^{\prime \prime}=0$ along $M_{0}$. Therefore we may proceed in the standard way as in $[10,11]$ for $U^{\prime \prime}$ and we get

$$
\left\|A U^{\prime \prime}\right\|^{2}+\left\|B U^{\prime \prime}\right\|^{2} \geq c\left(L\left(U^{\prime \prime}\right)+\int_{M_{\sigma}}\left|U^{\prime \prime}\right|^{2} d S\right) .
$$

A typical term of $E\left(U^{\prime}, U^{\prime \prime}\right)$ looks like

$$
\left(L_{k} U_{l}^{k K}, L_{n} U_{l}^{n K}\right)-\left(\bar{L}_{n} U_{l}^{k K}, \bar{L}_{k} U_{l}^{n K}\right),
$$


where $n \notin K$ and $1 \leq k \leq n-1$. Since $U_{l}^{k K}=0$ on $M_{0}$ and $U_{l}^{n K}=0$ on $M_{\sigma}$, we can perform the integration by parts and we get

$$
\begin{aligned}
\left(L_{k} U_{l}^{k K}, L_{n} U_{l}^{n K}\right)= & \left(\bar{L}_{n} U_{l}^{k K}, \bar{L}_{k} U_{l}^{n K}\right)-\left(e_{k} \bar{L}_{n} U_{l}^{k K}, U_{l}^{n K}\right) \\
& +\left(\bar{e}_{n} L_{k} U_{l}^{k K}, U_{l}^{n K}\right)+\left(\left[L_{k}, \bar{L}_{n}\right] U_{l}^{k K}, U_{l}^{n K}\right) .
\end{aligned}
$$

By integration by parts if necessary, the second and the third term of the right side of (5.24) are bounded by $\sigma L\left(U^{\prime}\right)+C \sigma^{-1}\|U\|^{2}$. If we write

$$
\left(\left[L_{k}, \bar{L}_{n}\right] U_{l}^{k K}, U_{l}^{n K}\right)=\sum_{i=1}^{n}\left(c_{k n}^{i} L_{i} U_{l}^{k K}, U_{l}^{n K}\right)+\sum_{i=1}^{n}\left(d_{k n}^{i} \bar{L}_{i} U_{l}^{k K}, U_{l}^{n K}\right),
$$

where $c_{k n}^{i}=\omega^{i}\left(\left[L_{k}, \bar{L}_{n}\right]\right)$ and $d_{k n}^{i}=\bar{\omega}^{i}\left(\left[L_{k}, \bar{L}_{j}\right]\right)$, then when $i<n$, the each term of right is dominated by $\sigma L\left(U^{\prime}\right)+\sigma^{-1}\|U\|^{2}$, by applying integration by parts if necessary. If $i=n$, then $\left(d_{k n}^{n} \bar{L}_{n} U_{l}^{k K}, U_{l}^{n K}\right)$ is bounded by $\sigma L\left(U^{\prime}\right)+\sigma^{-1}\|U\|^{2}$. The remaining term $\left(c_{k n}^{n} L_{n} U_{l}^{k K}, U_{l}^{n K}\right)$ can be handled by using Lemma 5.5. Therefore we conclude that

$$
\left|E\left(U^{\prime}, U^{\prime \prime}\right)\right| \leq C\left(\sigma L\left(U^{\prime}\right)+\sigma^{-1}\|U\|^{2}\right) .
$$

Let $\lambda \in C^{\infty}\left(\tilde{W}\left(x_{0}\right)\right)$ with $|\lambda| \leq 1$, and for $f \in C^{\infty}\left(W\left(x_{0}\right)\right)$, we define

$$
\|f\|_{\lambda}^{2}=\int_{W\left(x_{0}\right)}|f|^{2} e^{-\lambda} d V .
$$

Combining (5.22)-(5.26), we conclude that

$$
\begin{aligned}
\|A U\|^{2}+\|B U\|^{2} \geq c_{1} & \left(L\left(U^{\prime \prime}\right)+\int_{M_{\sigma}}\left|U^{\prime \prime}\right|^{2} d V\right) \\
& +\frac{1}{3}\left(\left\|A U^{\prime}\right\|_{\lambda}^{2}+\left\|B U^{\prime}\right\|_{\lambda}^{2}\right) \\
& -C \sigma L\left(U^{\prime}\right)-C \sigma^{-1}\|U\|^{2},
\end{aligned}
$$

because $e^{-\lambda} \geq 1 / 3$.

Now let us estimate $\left\|A U^{\prime}\right\|_{\lambda}^{2}+\left\|B U^{\prime}\right\|_{\lambda}^{2}$. As in (4.2.3) of [11], we get

$$
\left\|A U^{\prime}\right\|_{\lambda}^{2}+\left\|B U^{\prime}\right\|_{\lambda}^{2}=L_{\lambda}\left(U^{\prime}\right)+\sum_{l=1}^{n} \sum_{j, k=1}^{n-1}\left[\left(L_{k} U_{l}^{k n}, L_{j} U_{l}^{j n}\right)_{\lambda}-\left(\bar{L}_{j} U_{l}^{k n}, \bar{L}_{k} U_{l}^{j n}\right)_{\lambda}\right],
$$

where $L_{\lambda}\left(U^{\prime}\right)=\sum_{l=1}^{n} \sum_{\substack{|J|=q \\ n \in J}} \sum_{k=1}^{n-1}\left\|\bar{L}_{k} U_{l}^{J}\right\|_{\lambda}^{2}+\sum_{l=1}^{n} \sum_{\substack{|J|=q \\ n \in J}}\left\|L_{n} U_{l}^{J}\right\|_{\lambda}^{2}$.

With the notation $\delta_{k}=e^{\lambda} L_{k} e^{-\lambda}, 1 \leq k \leq n-1$, we have

$$
\begin{aligned}
\left(\delta_{k} U_{l}^{k n}, \delta_{j} U_{l}^{j n}\right)_{\lambda}= & \left(L_{k} U_{l}^{k n}, L_{j} U_{l}^{j n}\right)_{\lambda} \\
& +\mathcal{O}\left(\sigma^{-1 / 4}\left\|(L \lambda) U^{\prime}\right\|^{2}+\sigma^{1 / 4} \sum_{k=1}^{n-1}\left\|L_{k} U_{l}^{k n}\right\|^{2}\right),
\end{aligned}
$$


where $\left\|(L \lambda) U^{\prime}\right\|^{2}=\left\|\sum_{k=1}^{n-1}\left(L_{k} \lambda\right) U_{l}^{k n}\right\|^{2}$. By the standard integration by parts method, we obtain that

$$
\left\|L_{k} U_{l}^{k n}\right\|^{2}=\left\|\bar{L}_{k} U_{l}^{k n}\right\|^{2}-\int_{M_{0}} d_{k k}^{n}\left|U_{l}^{k n}\right|^{2} d S+\mathcal{O}\left(\sigma L\left(U^{\prime}\right)+\sigma^{-1}\left\|U^{\prime}\right\|^{2}\right) .
$$

As in (5.24), we can write, for $1 \leq j, k \leq n-1$, as

$$
\begin{aligned}
\left(\delta_{k} U_{l}^{k n}, \delta_{j} U_{l}^{j n}\right)_{\lambda}= & \left(\bar{L}_{j} U_{l}^{k n}, \bar{L}_{k} U_{l}^{j n}\right)_{\lambda}-\left(e_{k} \bar{L}_{j} U_{l}^{k n}, U_{l}^{j n}\right)_{\lambda} \\
& +\left(\bar{e}_{j} \delta_{k} U_{l}^{k n}, U_{l}^{j n}\right)_{\lambda}+\left(\left[\delta_{k}, \bar{L}_{j}\right] U_{l}^{k n}, U_{l}^{j n}\right)_{\lambda} .
\end{aligned}
$$

By integration by parts, if necessary, the second and third terms of (5.31) are bounded by $C \sigma L\left(U^{\prime}\right)+C \sigma^{-1}\left\|U^{\prime}\right\|^{2}$. To estimate $\left(\left[\delta_{k}, \bar{L}_{j}\right] U_{l}^{k n}, U_{l}^{j n}\right)_{\lambda}$, we write

$$
\begin{aligned}
\left(\left[\delta_{k}, \bar{L}_{j}\right] U_{l}^{k n}, U_{l}^{j n}\right)_{\lambda}= & \sum_{i=1}^{n}\left(c_{k j}^{i} L_{i} U_{l}^{k n}, U_{l}^{j n}\right)_{\lambda}+\sum_{i=1}^{n}\left(d_{k j}^{i} \bar{L}_{i} U_{l}^{k n}, U_{l}^{j n}\right)_{\lambda} \\
& +\left(\bar{L}_{j}\left(L_{k} \lambda\right) U_{l}^{k n}, U_{l}^{j n}\right)_{\lambda},
\end{aligned}
$$

where $c_{k j}^{i}=\omega^{i}\left(\left[L_{k}, \bar{L}_{j}\right]\right)$, and $d_{k j}^{i}=\bar{\omega}^{i}\left(\left[L_{k}, \bar{L}_{j}\right]\right)$.

If $i<n$, then $\left(d_{k j}^{i} \bar{L}_{i} U_{l}^{k n}, U_{l}^{j n}\right)_{\lambda}$ is bounded by $\sigma L\left(U^{\prime}\right)+C \sigma^{-1}\left\|U^{\prime}\right\|^{2}$. By integration by parts, we can write

$$
\left(c_{k j}^{i} L_{i} U_{l}^{k n}, U_{l}^{j n}\right)_{\lambda}=\left(c_{k j}^{i}\left(L_{i} \lambda\right) U_{l}^{k n}, U_{l}^{j n}\right)_{\lambda}+\mathcal{O}\left(\sigma L\left(U^{\prime}\right)+\sigma^{-1}\left\|U^{\prime}\right\|^{2}\right) .
$$

If $i=n$, then $\left(c_{k j}^{n} L_{n} U_{l}^{k n}, U_{l}^{j n}\right)_{\lambda}$ is bounded by $\sigma L\left(U^{\prime}\right)+C \sigma^{-1}\left\|U^{\prime}\right\|^{2}$. By integration by parts again, we can write

$$
\begin{aligned}
\left(d_{k j}^{n} \bar{L}_{n} U_{l}^{k n}, U_{l}^{j n}\right)_{\lambda}= & \left(d_{k j}^{n}\left(\bar{L}_{n} \lambda\right) U_{l}^{k n}, U_{l}^{j n}\right)_{\lambda}-\int_{M_{0}} d_{k j}^{n} U_{l}^{k n} \bar{U}_{l}^{j n} e^{-\lambda} d S \\
& +\mathcal{O}\left(\sigma L\left(U^{\prime}\right)+\sigma^{-1}\left\|U^{\prime}\right\|^{2}\right) .
\end{aligned}
$$

Combining (5.28)-(5.34), we obtain that

$$
\begin{aligned}
\left\|A U^{\prime}\right\|_{\lambda}^{2}+\left\|B U^{\prime}\right\|_{\lambda}^{2} \geq & \sum_{j, k=1}^{n-1}\left[\left(\bar{L}_{j}\left(L_{k} \lambda\right) U_{l}^{k n}, U_{l}^{j n}\right)_{\lambda}+\sum_{i=1}^{n-1}\left(c_{k j}^{i}\left(L_{i} \lambda\right) U_{l}^{k n}, U_{l}^{j n}\right)_{\lambda}\right] \\
& +\sum_{j, k=1}^{n-1}\left(d_{k j}^{n}\left(\bar{L}_{n} \lambda\right) U_{l}^{k n}, U_{l}^{j n}\right)_{\lambda} \\
& -\sum_{j, k=1}^{n-1} \int_{M_{0}}\left(d_{k j}^{n} U_{l}^{k n} \bar{U}_{l}^{j n}+C_{1} \sigma^{1 / 4} d_{k k}^{n}\left|U_{l}^{k n}\right|^{2}\right) e^{-\lambda} d S \\
& +c L\left(U^{\prime}\right)-C_{1} \sigma^{-1 / 4}\left\|(L \lambda) U^{\prime}\right\|^{2}-C \sigma^{-1}\left\|U^{\prime}\right\|^{2} .
\end{aligned}
$$

With the notation

$$
\partial \bar{\partial} \lambda=\sum_{j, k=1}^{n-1} \lambda_{j k} \omega^{j} \wedge \bar{\omega}^{k}
$$


the first sum of the right hand side of (5.35) is equal to $\sum_{j, k=1}^{n-1}\left(\lambda_{j k} U_{l}^{j n}, U_{l}^{k n}\right)_{\lambda}$. Now suppose that $|\lambda| \leq 1$ on $\tilde{W}\left(x_{0}\right)$. Let $\chi(t)$ denote the function $\sigma^{1 / 4} / 3 C_{1} e^{t}$ and set $\phi=\chi(\lambda)$. Then $\chi^{\prime \prime}(t) \geq C_{1} \sigma^{-1 / 4} \chi^{\prime}(t)^{2}$, and we get

$$
\begin{aligned}
\sum_{j, k=1}^{n-1} \phi_{j k} t_{j} \bar{t}_{k} & =\chi^{\prime}(t) \sum_{j, k=1}^{n-1} \lambda_{j k} t_{j} \bar{t}_{k}+\chi^{\prime \prime}(t)\left|\sum_{k=1}^{n-1}\left(L_{k} \lambda\right) t_{k}\right|^{2} \\
& \geq \frac{\sigma^{1 / 4}}{9 C_{1}} \sum_{j, k=1}^{n-1} \lambda_{j k} t_{j} \bar{t}_{k}+C_{1} \sigma^{-1 / 4} \chi^{\prime}(t)^{2}\left|\sum_{k=1}^{n-1}\left(L_{k} \lambda\right) t_{k}\right|^{2} .
\end{aligned}
$$

Since $M_{0}$ is pseudoconvex and $d_{k k}^{n}>d>0$ for $2 \leq k \leq n-1$, it follows that

$$
-\sum_{j, k=1}^{n-1} \int_{M_{0}}\left(d_{k j}^{n} U_{l}^{k n} \bar{U}_{l}^{j n}+C_{1} \sigma^{1 / 4} d_{k k}^{n}\left|U_{l}^{k n}\right|^{2}\right) e^{-\lambda} d S \geq 0,
$$

provided that $\sigma$ is sufficiently small. Thus, if we replace $\lambda$ by $\phi$, then we conclude from (5.36) and (5.37) that

$$
\begin{aligned}
\left\|A U^{\prime}\right\|_{\phi}^{2}+\left\|B U^{\prime}\right\|_{\phi}^{2} \geq & \frac{\sigma^{1 / 4}}{9 C_{1}} \sum_{j, k=1}^{n-1}\left(\lambda_{j k} U_{l}^{j n}, U_{l}^{k n}\right)_{\phi} \\
& +\sum_{j, k=1}^{n-1}\left(d_{k j}^{n}\left(\bar{L}_{n} \lambda\right) U_{l}^{k n}, U_{l}^{j n}\right)_{\phi} \\
& +c L\left(U^{\prime}\right)-C \sigma^{-1}\left\|U^{\prime}\right\|^{2} .
\end{aligned}
$$

Now we take the family $\left\{\lambda_{\rho}\right\}_{\rho>0}$ of plurisubharmonic functions with maximal Hessian constructed in Theorem 4.5, and replace $\lambda$ in (5.38) by these functions. By Lemma 5.6 and the fact that $\tau(x, \delta) \approx \mu(x, \delta)$, we have, for $y=D_{x_{0}}(x) \in S_{\rho}$, that

$$
\begin{aligned}
& \mid\left(d_{11}^{n}\left(\bar{L}_{n} \lambda_{\rho}\right)(y) \mid \lesssim \sigma^{1 / 2} \mu\left(x, \delta_{0}\right)^{2} \mu\left(x, \rho \delta_{0}\right)^{-2},\right. \\
& \mid\left(d_{1 k}^{n}\left(\bar{L}_{n} \lambda_{\rho}\right)(y) \mid \lesssim \sigma^{1 / 2} \rho^{-1 / 2} \mu\left(x, \delta_{0}\right) \mu\left(x, \rho \delta_{0}\right)^{-1+\gamma}, \quad 2 \leq k \leq n-1 .\right.
\end{aligned}
$$

Also, it follows from (4.27) and (4.28) that

$$
\mid\left(d_{j, k}^{n}\left(\bar{L}_{n} \lambda_{\rho}\right)(y) \mid \lesssim \sigma^{1 / 2} \rho^{-1}, \quad 2 \leq j, k \leq n-1 .\right.
$$

By virtue of Theorem 4.5 it follows that (assuming that we first take $\rho_{0} \leq \sigma^{2 m}$ for sufficiently small $\sigma$ ) there is, for each $0<\rho \leq \sigma^{2 m}, \lambda=\lambda_{\rho}$ such that

$$
\sum_{j, k=1}^{n-1} \lambda_{j, k}(y) U_{l}^{j n}(y) \bar{U}_{l}^{j n}(y) \approx \sum_{k=1}^{n-1}\left|U^{k n}(y)\right|^{2} \mu_{k}^{2}(x)\left(\mu_{k}^{\rho}(x)\right)^{-2}, \quad y \in S_{\rho},
$$

where $\mu_{k}(x)$ and $\mu_{k}^{\rho}(x)$ are defined in (4.33) and (4.34), respectively. 
Combining (5.38)-(5.42), we then have that

$$
\begin{aligned}
\left\|A U^{\prime}\right\|^{2}+\left\|B U^{\prime}\right\|^{2} & \geq c \sigma^{1 / 2} \sum_{j, k=1}^{n-1}\left(\lambda_{j k} U_{l}^{j n}, U_{l}^{k n}\right)+c L\left(U^{\prime}\right) \\
& \geq c\left(\sigma^{1 / 2} \rho^{-2 / m}\left\|U^{\prime}\right\|^{2}+L\left(U^{\prime}\right)\right)
\end{aligned}
$$

because $\mu\left(x, \delta_{0}\right)^{2} \mu\left(x, \rho \delta_{0}\right)^{-2} \gtrsim \rho^{-1 / m}$ by (3.34). Then, by the theorem of Catlin [2], the subelliptic estimates of order $1 / m$ holds for $U^{\prime}$ and hence we get

$$
\sigma^{1 / 2}\left\|U^{\prime}\right\|_{1 / m}^{2}+L\left(U^{\prime}\right) \leq C\left(\left\|A U^{\prime}\right\|^{2}+\left\|B U^{\prime}\right\|^{2}\right)+\left\|U^{\prime}\right\|^{2} .
$$

Combining (5.21), (5.27) and (5.43), we conclude that

$$
\|S U\|^{2}+\left\|T^{*} U\right\|^{2} \geq c\left(\left.\sigma^{1 / 2}\left|\left\|U^{\prime}\right\|_{1 / m}^{2}+L(U)+\int_{M_{\sigma}}\right| U^{\prime \prime}\right|^{2} d S\right)-C \sigma^{-1}\|U\|^{2} .
$$

If $n=2$, then $U^{\prime \prime}=0$ on $M_{\sigma}$, and if $n \geq 3$, then we have at least one positive eigenvalue. In this case, we apply Lemma 5.3 for $f$ replaced by $U^{\prime \prime}$ and get

$$
\sigma^{1 / 2}\left\|\mid U^{\prime \prime}\right\|_{1 / 2}^{2} \leq C L\left(U^{\prime \prime}\right) \text {. }
$$

Combining (5.4), (5.44) and (5.45), we conclude that

$$
\sigma^{-3}\|U\|^{2}+L(U)+\sigma^{1 / 2}\|\| U \|_{1 / m}^{2} \leq C\left(\|S U\|^{2}+\left\|T^{*} U\right\|^{2}\right),
$$

for all $U \in \mathcal{B}^{q}\left(S_{\varepsilon, \sigma} ; A\right)$, provided $\sigma$ is sufficiently small.

For the estimates of the non-tangential derivatives of $U$, we note that $L_{n}^{A}=\partial / \partial y_{2 n}+X$, where $X=\sum_{j=1}^{2 n-1} b_{j}(y) \partial / \partial y_{j}$. Therefore a standard argument yields the inequality

$$
\left.\left\|\frac{\partial f}{\partial y_{2 n}}\right\|\right|_{-1+1 / m} ^{2} \leq C\left(1+\sum_{j=1}^{2 n-1}\left|b_{j}\right|_{\tilde{W}\left(x_{0}\right), 2 n+3}^{2}\right)\left(\left\|\left|f\left\|\left.\right|_{1 / m} ^{2}+\right\| \bar{L}_{n} f\left\|^{2}+\right\| f \|^{2}\right)\right.\right.
$$

for all $f \in C_{0}^{\infty}\left(\tilde{W}\left(x_{0}\right)\right)$. This inequality can be applied with $f=U_{l}^{J}$ to obtain (5.1) from (5.46). This completes the proof of Theorem 5.1.

We now define Sobolev spaces for sections of $\Gamma^{0, q}\left(S_{\varepsilon, \sigma} ; A\right)$. Recall that the open sets $B_{b}\left(x_{0}\right)$ satisfy (4.3) and (4.4) for each $x_{0} \in M$. Choose a set $T_{\sigma}=\left\{x_{i}^{\sigma} \in M ; i \in I\right\}$ such that the sets $B_{c \sigma / 2}\left(x_{i}^{\sigma}\right), i \in I$, cover $S_{\varepsilon, \sigma}$, and such that no two points $x_{i}^{\sigma}$ and $x_{j}^{\sigma}$ satisfy $\left|x_{i}^{\sigma}-x_{j}^{\sigma}\right| \leq c \sigma / 4$, where | | is the distance function on $S_{\varepsilon, \sigma}$. It follows that the sets $W\left(x_{i}^{\sigma}\right), i \in I$, cover $S_{\varepsilon, \sigma}$ and that there exists an integer $\tilde{N}$ such that no point of $S_{\varepsilon, \sigma}$ lies in more than $\tilde{N}$ open sets $W\left(x_{i}^{\sigma}\right)$. Furthermore, there exist functions $\zeta_{i}, \zeta_{i}^{\prime}$ (that are independent of $\left.y_{2 n}\right) \in C_{0}^{\infty}\left(W\left(x_{i}^{\sigma}\right)\right)$ such that $\sum_{i \in I} \zeta_{i}^{2} \equiv 1$, and if $x \in \operatorname{supp} \zeta_{i}$, then

$$
\zeta_{i}^{\prime} \equiv 1 \text { in } B_{c^{\prime} \sigma}(x),
$$

and that both $\zeta_{i}$ and $\zeta_{i}^{\prime}$ satisfy

$$
\left|\zeta_{i}\right|_{k, W\left(x_{i}^{\sigma}\right)}+\left|\zeta_{i}^{\prime}\right|_{k, W\left(x_{i}^{\sigma}\right)} \leq C_{k} \sigma^{-k} .
$$


Now, let $F$ be any section of $\Gamma^{0, q}\left(S_{\varepsilon, \sigma} ; A\right)$. We define

$$
\|F\|_{k, A}^{2}=\sum_{i \in I}\left\|\zeta_{i} F\right\|_{k, A, W\left(x_{i}^{\sigma}\right)}^{2}
$$

where

$$
\left\|\zeta_{i} F\right\|_{k, A, W\left(x_{i}^{\sigma}\right)}^{2}=\sum_{l=1}^{n} \sum_{|J|=q}\left\|\zeta_{i} F_{l}^{J}\right\|_{k, W\left(x_{i}^{\sigma}\right)}^{2},
$$

and $F=\sum_{l=1}^{n} \sum_{|J|=q} F_{l}^{J} \bar{\omega}_{A}^{J} \cdot L_{j}^{A}$ is the decomposition of $F$ in terms of the frame of $W\left(x_{i}^{\sigma}\right)$. Moreover, the Sobolev norm $\|\quad\|_{k, W\left(x_{i}^{\sigma}\right)}$ is taken with respect to the $y$-coordinates of $W\left(x_{i}^{\sigma}\right)$. We define $H_{k}^{0, q}\left(S_{\varepsilon, \sigma} ; T_{A}^{1,0}\right)$ to be the set of all sections $F$ of $\Gamma^{0, q}\left(S_{\varepsilon, \sigma} ; A\right)$ for which $\|F\|_{k, A}<\infty$. If we define $L_{q}^{2}\left(S_{\varepsilon, \sigma} ; T_{A}^{1,0}\right)$ to be the set of all $F \in \Gamma^{0, q}\left(S_{\varepsilon, \sigma} ; A\right)$ such that $\|F\|^{2}<\infty$, then it is obvious that the norms $\|\quad\|$ and \|\|$_{0, A}$ are equivalent on $L_{q}^{2}\left(S_{\varepsilon, \sigma} ; T_{A}^{1,0}\right)$. Since $\mathcal{A}\left(S_{\varepsilon, \sigma}\right) \subset \Gamma^{0,1}\left(S_{\varepsilon, \sigma} ; 0\right)$, we define $\|A\|_{k}=\|A\|_{k, 0}$ for $A \in \mathcal{A}\left(S_{\varepsilon, \sigma}\right)$, and $H_{k}\left(S_{\varepsilon, \sigma} ; \mathcal{A}\right)$ to be the set of $A \in \mathcal{A}\left(S_{\varepsilon, \sigma}\right)$ such that $\|A\|_{k}<\infty$.

We want to get an estimate in global form. Define $Q(U, U)=\left\|T^{*} U\right\|^{2}+\|S U\|^{2}$. By using the partition of unity as defined above satisfying (5.47) and (5.48), and from the estimates in Theorem 5.1, we obtain the following

COROLLARY 5.7. There exist a fixed small $\sigma$ and a constant $\varepsilon_{1}>0$ such that for all $\varepsilon, 0<\varepsilon<\varepsilon_{1}$ and all $U \in \operatorname{Dom}\left(T^{*}\right) \cap \operatorname{Dom}(S)$,

$$
\|U\|^{2} \leq C Q(U, U) \text {. }
$$

Now let us fix $\sigma>0$ satisfying Corollary 5.7, and set $W\left(x_{0}\right)=W_{\sigma}\left(x_{0}\right)$. Using Theorem 5.1 and the standard "bootstrap" method, we can get regularity estimates for the linearized equation. The proof is similar to that in Section 9 of [5]. Here we use $1 / \mathrm{m}$ subelliptic estimates instead of $1 / 2$ subellitic estimates. Set $\square=D_{q} D_{q}^{*}+D_{q+1}^{*} D_{q+1}$. In the sequel, we assume that $A$ satisfies

$$
\|A\|_{2 n+3} \leq \varepsilon_{0} .
$$

THEOREM 5.8. Suppose that $U$ is the solution of $\square U=G$, where $G \in H_{k}^{0, q}\left(S_{\varepsilon} ; T_{A}^{1,0}\right)$ for all $k>0$. Then

$$
\left\|D_{q}^{*} U\right\|_{k}+\left\|D_{q+1} U\right\|_{k} \lesssim\|G\|_{k}+\left(1+\|A\|_{k+2}\right)\|G\|_{n+2} .
$$

Now set $E=D_{q+1}^{*} D_{q+1} U$. Then we have the following estimates for the error term $E$ [5, Theorem 10.3].

THEOREM 5.9. Suppose that $\square U=G$, where $D_{q+1} G=0$ and $G \in H_{k}^{0, q}\left(S_{\varepsilon} ; T_{A}^{1,0}\right)$ for all $k$. Then $E=D_{q+1}^{*} D_{q+1} U$ satisfies

$$
\begin{aligned}
\|E\|_{k-1} \lesssim & \|G\|_{k}\left\|F^{A}\right\|_{n+1}+\|G\|_{n+1}\left\|F^{A}\right\|_{k} \\
& +\left(1+\|A\|_{k+2}\right)\|G\|_{n+1}\left\|F^{A}\right\|_{n+1} .
\end{aligned}
$$

Note that $F^{A}$ is $D_{3}$-closed. Since $q=2$, we immediately obtain 
COROLLARY 5.10. If $U$ is the solution with respect to $\mathcal{L}^{A}$ of $\square U=F^{A}$, then $V^{\prime}=$ $D_{2}^{*} U$ satisfies for all $k=n+1, n+2, \ldots$

$$
\left\|D_{2} V^{\prime}-F^{A}\right\|_{k} \lesssim\left\|F^{A}\right\|_{k+1}\left\|F^{A}\right\|_{n+1}+\|A\|_{k+3}\left\|F^{A}\right\|_{n+1}^{2} .
$$

6. Extension of CR structures. In this section we will prove Theorem 1.1 and Theorem 1.4, using the estimates in Section 5. First, we describe the nonlinear extension operator. For details, one can refer to Section 11 of [5].

If $A \in \mathcal{A}\left(S_{\varepsilon, \sigma}\right)$ is sufficiently small and if we set $P_{A}(\bar{L})=\bar{L}+A(\bar{L})$, then $\overline{\mathcal{L}}_{A}=$ $\left\{P_{A}(\bar{L}) ; \bar{L} \in \overline{\mathcal{L}}\right\}$. If we set $Q_{A}(\omega)=\omega-A^{*} \omega$, then $\Lambda_{A}^{1,0}=\left\{Q_{A}(\omega) ; \omega \in \Lambda^{1,0}(\mathcal{L})\right\}$. We define a nonlinear operator $\Phi: \mathcal{A}\left(S_{\varepsilon, \sigma}\right) \rightarrow \Gamma^{0,2}\left(S_{\varepsilon, \sigma}\right)$ by

$$
\Phi(A)\left(\bar{L}^{\prime}, \bar{L}^{\prime \prime}, \omega\right)=Q_{A}(\omega)\left(\left[P_{A}\left(\bar{L}^{\prime}\right), P_{A}\left(\bar{L}^{\prime \prime}\right)\right]\right) .
$$

Obviously, if $\Phi(A)=0$, then $\mathcal{L}_{A}$ is an integrable almost complex structure on $S_{\varepsilon, \sigma}$.

Note that there is a natural map $\mathcal{P}_{A}: \Gamma_{A}^{0,2} \rightarrow \Gamma^{0,2}$ defined by

$$
\left(\mathcal{P}_{A} B\right)\left(\bar{L}_{1}, \bar{L}_{2}, \omega\right)=B\left(P_{A}\left(\bar{L}_{1}\right), P_{A}\left(\bar{L}_{2}\right), Q_{A}(\omega)\right), \quad B \in \Gamma_{A}^{0,2} .
$$

Therefore it follows from the definition of $F^{A}$ in $(2.5)$ that $\Phi(A)=\mathcal{P}_{A}\left(F^{A}\right)$. We also note that if $d$ and $A$ are small sections of $\mathcal{A}$ on $S_{\varepsilon, \sigma}$, then there exist sections $\Delta_{A, d}^{+}$and $\Delta_{A, d}^{-}$of $\Lambda_{A}^{0,1} \otimes T_{A}^{1,0}$ and $\Lambda_{A}^{0,1} \otimes T_{A}^{0,1}$, respectively, such that

$$
P_{A+d}(\bar{L})=P_{A}(\bar{L})+\Delta_{A, d}^{+}\left(P_{A}(\bar{L})\right)+\Delta_{A, d}^{-}\left(P_{A}(\bar{L})\right) .
$$

Similarly, there exist sections $\delta_{A, \delta}^{+}$and $\delta_{A, \delta}^{-}$of $\operatorname{Hom}\left(\Lambda_{A}^{1,0}, \Lambda_{A}^{1,0}\right)$ and $\operatorname{Hom}\left(\Lambda_{A}^{1,0}, \Lambda_{A}^{0,1}\right)$, respectively, such that

$$
Q_{A+d}(\omega)=Q_{A}(\omega)-\delta_{A, d}^{+}\left(Q_{A}(\omega)\right)-\delta_{A, d}^{-}\left(Q_{A}(\omega)\right)
$$

Then it follows that $\Delta_{A}^{ \pm}(d)=\Delta_{A, d}^{ \pm}$both depend linearly on $d$ and that the coefficients depend smoothly on $A$, and the mapping $d \rightarrow \Delta_{A}(d)=\Delta_{A}^{+}(d)+\Delta_{A}^{-}(d)$ is invertible. Then $\Phi^{\prime}(A)(d)$, as an element of $\Gamma^{0,2}$, satisfies

$$
\Phi^{\prime}(A)(d)=\left(\mathcal{P}_{A} \circ D_{2}^{A} \circ \Delta_{A}^{+}\right)(d)-\mathcal{P}_{A}\left(h_{A}(d)\left(F^{A}\right)\right),
$$

where $h_{A}(d): T_{A}^{1,0} \rightarrow T_{A}^{1,0}$ denotes the adjoint of $\delta_{A}^{+}(d): \Lambda_{A}^{1,0} \rightarrow T_{A}^{1,0}$. Since $\Phi(A)=$ $\mathcal{P}_{A}\left(F^{A}\right)$, we let $U_{A}$ be the solution of $\square U_{A}=-F^{A}$, and then set $V_{A}=\left(D_{2}^{A}\right)^{*} U_{A}$ and $d_{A}=\Delta_{A}^{-1}\left(V_{A}\right)$. Using the error estimates in Theorem 5.9 and Corollary 5.10, we then obtain the following good estimates for the approximate solution of $\Phi(A)+\Phi^{\prime}(A)(d)=0$.

THEOREM 6.1. Suppose that $A \in H_{k}\left(S_{\varepsilon, \sigma}, \mathcal{A}\right)$ for all $k$. Then there exists $d_{A} \in$ $H_{k}\left(S_{\varepsilon, \sigma}, \mathcal{A}\right)$ for all $k$ such that if $k \geq n+2$,

$$
\begin{aligned}
& \left\|d_{A}\right\|_{k} \lesssim\|\Phi(A)\|_{k}+\|A\|_{k+2}\|\Phi(A)\|_{n+2}, \\
& \left\|\Phi(A)+\Phi^{\prime}(A)\left(d_{A}\right)\right\|_{k-1} \lesssim\|\Phi(A)\|_{k}\|\Phi(A)\|_{n+2}+\|A\|_{k+2}\|\Phi(A)\|_{n+2}^{2} .
\end{aligned}
$$


Note that properties (6.3) and (6.4) of the nonlinear operator $\Phi$ are the crucial ingredients in the application of simplified Nash-Moser iteration process [15].

We recall that $F^{A}$ vanishes to infinite order along $M_{0}$ (in $x$-coordinates!). This can be stated in $y$-coordinates as follows. The proof is similar to that of Lemma 6.2 in [5].

LEMMA 6.2. Suppose that there exists a section $F \in \Gamma^{0,2}\left(\bar{\Omega}^{+}\right)$, where $\bar{\Omega}^{+}=\{(x, t) \in$ $\Omega ; 0 \leq t<1\}$ such that $F$ and all its derivatives vanish to infinite order along $M$. Then for all $k, N=0,1,2, \ldots$, and all $x_{0} \in M$,

$$
\left|F^{0}\right|_{k, W\left(x_{0}\right)} \leq C_{k, N} \varepsilon^{N} \varphi\left(x_{0}\right)^{N}
$$

where $F^{0}$ means that $F$ is written out in $W\left(x_{0}\right)$ according to the frames $L_{1}^{0}, \ldots, L_{n}^{0}$, $\omega_{0}^{1}, \ldots, \omega_{0}^{n}$ of $\mathcal{L}^{0}$.

We can now prove the main theorems of this paper:

Proof of TheOrem 1.1. We will show that $\|\Phi(0)\|_{D}<b$ for the small $b>0$ and the integer $D$, which are appeared in the variant of Nash-Moser theorem [15]. The rest properties for the $\Phi(A)$ in the hypothesis of Nash-Moser theorem can be proved using the relations in (6.3) and (6.4), and the estimates for $\square$ operator in Section 5.

Note that (5.48) and (6.5) imply that for each $i \in I$,

$$
\left\|\zeta_{i} F^{0}\right\|_{k, 0}^{2} \leq C_{k, N} \varepsilon^{N} \varphi\left(x_{i}^{\sigma}\right)^{N},
$$

where $\zeta_{i}$ 's are defined before (5.47). After summing up over $x_{i}^{\sigma}$, we get

$$
\left\|F^{0}\right\|_{k, 0, \Phi}^{2} \leq C_{k, N} \sum_{i \in I} \varphi\left(x_{i}^{\sigma}\right)^{N} \varepsilon^{N} .
$$

Since the choice of points that was made before (5.48) shows that the balls $B_{c \sigma / 8}\left(x_{i}^{\sigma}\right)$, $i \in I$, are all disjoint, we can obtain an upper bound on $N(l)$, which is defined to be the number of $i \in I$ such that $2^{-l-1} \leq \varphi\left(x_{i}^{\sigma}\right)<2^{-l}$. In fact, in terms of the $\langle,\rangle_{0}$-metric introduced at the end of Section 2, the volume of $B_{c \sigma / 8}\left(x_{i}^{\sigma}\right)$ is roughly bounded below by $\varepsilon^{n+1} \sigma^{2 n-1+2 m} \varphi\left(x_{i}^{\sigma}\right)^{2 m(n+2)} \sim \varepsilon^{n+1} \sigma^{2 n-1+2 m} \cdot 2^{-2 l m(n+2)}$, and the $\langle,\rangle_{0}$-volume of the region in $S_{\varepsilon, \sigma}$ with $2^{-l-1} \leq \varphi(x) \leq 2^{-l}$ is roughly bounded above by $\varepsilon \sigma^{2 m} \cdot 2^{-2 m l}$. Thus, we conclude that

$$
N(l) \lesssim \varepsilon^{-n} \sigma^{-(2 n-1)} 2^{2 m l(n+1)} .
$$

Thus (6.6) and (6.7) imply that if $N=2 m l(n+1)+1$, then

$$
\left\|\Phi\left(A_{0}\right)\right\|_{k}=\left\|F^{0}\right\|_{k, 0} \lesssim C_{k} \cdot \varepsilon
$$

for sufficiently small $\varepsilon$. In particular, if we set $k=D$ and choose $\varepsilon$ to be sufficiently small, then it follow that $\|\Phi(A)\|_{D}<b$.

Proof of COROllary 1.3. Clearly, $\bar{M} \subset b D$ is a CR manifold satisfying all the conditions of Theorem 1.1. Therefore we can extend the given CR structure on $\bar{M}$ to the outside of $D$ by Theorem 1.1. Then, by virtue of Theorem 2.2, the extended CR structure can 
be patched smoothly with the given complex structure on $D$. Therefore Corollary 1.3 follows from the Newlander-Nirenberg theorem.

\section{REFERENCES}

[ 1] T. AKAHORI, A new approach to the local embedding theorem of CR structures for $n \geq 4$, Mem. Amer. Math. Soc. 67 (1987).

[2] D. Catlin, Subelliptic estimates for the $\bar{\partial}$-Neumann problem on pseudoconvex domains, Ann. of Math. (2) 126 (1987), 131-191.

[ 3 ] D. CATlin, A Newlander-Nirenberg theorem for manifolds with boundary Michigan, Math. J. 35 (1988), 233-240.

[4] D. Catlin, Estimates of invariant metrics on pseudoconvex domains of dimension two, Math. Z. 200 (1989), 429-466.

[ 5 ] D. CATlin, Sufficient conditions for the extension of CR structures, J. Geom. Anal. 4 (1994), 467-538.

[ 6] S. CHO, Extension of complex structures on weakly pseudoconvex compact complex manifolds with boundary, Math. Z. 211 (1992), 105-120.

[ 7 ] S. CHO, Boundary behavior of the Bergman kernel function on some pseudoconvex domains in $\boldsymbol{C}^{n}$, Trans. Amer. Math. Soc. 345 (1994), 803-817.

[ 8 ] S. Cho, Extension of CR-structures on three dimensional pseudoconvex CR manifolds, Nagoya Math. J. 152 (1998), 1-35.

[ 9 ] J. P. D’Angelo, Real hypersurfaces, order of contact, and applications, Ann. of Math. (2) 115 (1982), 615637.

[10] L. HörmANDER, $L^{2}$ estimates and existence theorems for the $\bar{\partial}$-operator, Acta Math. 113 (1965), 89-152.

[11] L. Hörmander, An Introduction to Complex Analysis in Several Variables, O. Van Nostrand Co., Inc., Princeton, N.J.-Toronto, Ont.-London, (1966).

[12] H. Jacobowitz And F. Treves, Non-realizable CR structures, Invent. Math. 66 (1982), 231-249.

[13] M. KuRANisHi, Strongly pseudoconvex CR structures over small balls, Ann. of Math. (2) 115 (1982), 451500 .

[14] A. Newlander And L. Nirenberg, Complex Analytic coordinates in almost complex manifolds, Ann. of Math. (2) 65 (1957), 391-404.

[15] X. SAInT RAYMOnd, A simple Nash-Moser implicit function theorem, Enseign. Math. (2) 35 (1989), $217-$ 226.

[16] S. Webster, On the proof of Kuranishi's embedding theorem, Ann. Inst. H. Poincaré Anal. Non Linéaire 6 (1989), 183-207.

DEPARTMENT OF MATHEMATICS

SOGANG UNIVERSITY

SEOUL, 121-742

KOREA

E-mail address: shcho@sogang.ac.kr 